\title{
SMF/FSO integrated dual-rate reliable and energy efficient WDM optical access network for smart and urban communities
}

\author{
Amit Kumar Garg ${ }^{1}$. Sanjeev Kumar Metya ${ }^{2} \cdot$ Ghanshyam Singh $^{3} \cdot$ Vijay Janyani $^{3}$. \\ Moustafa H. Aly ${ }^{4}$ N. H. Zainol Abidin ${ }^{5}$
}

Received: 20 April 2021 / Accepted: 18 September 2021 / Published online: 13 October 2021

(C) The Author(s), under exclusive licence to Springer Science+Business Media, LLC, part of Springer Nature 2021

\begin{abstract}
To handle the massive high-speed internet traffic, free space optics (FSO) or single-mode fiber (SMF) based fiber optic communication is being used everywhere across the world. These technologies are capable of providing huge bandwidth and transmitting the data at very high speed with low energy consumption. FSO is a very convenient technology to quickly expand the legacy network in the adverse geographical areas. However, its link performance is highly dependent of inconsistent weather conditions. SMF based fiber optic link has a very low loss and its performance is almost independent on the weather conditions. Though, the installation and maintenance of fibers are quite complex and costly. Individually, FSO or SMF links have their limitations and have to be integrated to leverage their benefits. In this paper, we integrated FSO/SMF links and compared the performance of the proposed architecture which is capable of providing high-speed dual-rate data transmission. The proposed architecture transmits data over either FSO or SMF or both links simultaneously and has $100 \%$ more reliability against any one of the link failures. In case of operational link failure (FSO/SMF), data may be switched to the alternative working link (SMF/FSO), simply by tuning the transmitted signal by $50 \mathrm{GHz}$. The proposed architecture is also reliable against the optical line terminal transceiver (TRx) failure as each user located in the network can be served by two transceivers (1 Gbps and $10 \mathrm{Gbps}$ ). The proposed architecture also supports the wavelength division multiplexing overlay transmission for broadcasting the common signal to all the available users in the networks. The architecture reduces $\sim 27 \%$ of the energy consumption by utilizing the appropriate link of hybrid architecture and TRx according to weather conditions and traffic load. The integrated architecture looks attractive for providing energy-efficient, high speed, and reliable internet coverage to the areas where there is a difficulty of laying fibers and has frequent fiber faults. The architecture is useful for strengthening and boosting rural and urban development.
\end{abstract}

Keywords Free space optics · Integrated SMF/FSO - TWDM PON · Energy efficient optical network · Hybrid fiber FSO - Information and communication technology

Amit Kumar Garg

amitgroup1988@gmail.com

Extended author information available on the last page of the article 


\section{Introduction}

At present, the whole world including India is suffering from the ongoing global pandemic COVID-19 situation, and since its first known case, there is a rapid transition in the working style across various sectors across the globe including information technology, healthcare, education, e-Commerce, etc. Most of the organizations have adopted the work-fromhome (WFH) culture to maintain social distancing (Kramer and Kramer 2020). Various companies have adopted the WFH methodology as it allows them to reduce the significant capital expenditure (CAPEX) and operational expenditure (OPEX). However, due to WFH working culture adoption, there is a huge broadband traffic growth to support various highspeed internet applications and a large number of users. To maintain the WFH culture, physical working infrastructure is being converted to the digital infrastructure, i.e., information and communication technology (ICT) infrastructure. In this regard, lots of countries are pushing the development of smart rural and urban communities by strengthening the smart ICT infrastructure that can support the enormous bandwidth demand with the least adverse effects such as low energy consumption and high service reliability with optimum resource utilization (Mohanty et al. 2016).

For transmitting the signals at very high data rates and supporting, the ICT architecture, optical communication is the most widely adopted technology across the globe (Yamamoto et al. 1988). There are two popular ways of transmitting the optical signals; one is by setting up the SMF-based fiber optics links (Chae and Tucker 2004) and another is by setting up the FSO links (Chan 2006; Khalighi and Uysal 2014; Alkholidi and Altowij 2014; Malik and Singh 2015). FSO has air as the medium while fiber optics has fiberglass as the medium for guiding the optical signals. Both schemes have their advantages and limitations. Fiber optic technology using SMF has attracted a lot of the service providers as practically it has a very low loss $(0.2 \mathrm{~dB} / \mathrm{km})$ in the third optical window and SMF link performance is almost independent on weather conditions. Through SMF, data can be transmitted with much low transmitted powers as compared to FSO. However, the installation of new fibers and maintenance of the existing optical fibers are quite complex, costly and may take a few days to set up new links or to find the faults in the existing link. Many times the fiber installation is not possible in adverse geographical areas containing rivers, bridges, and mountains etc. Also, for various places, due to the court stays or other legal issues, there may be a delay in fiber installation and one has to look for an alternative technology that is fast and more convenient to install i.e. FSO (Chae and Tucker 2004).

The major advantage of the FSO link is that it does not require any spectrum licensing (Chan 2006), and can be deployed in the existing infrastructure within a few hours. So, FSO is much more suitable for scaling up the existing architecture within a short span of time (Khalighi and Uysal 2014) especially in the hills, mountains, and river areas. The tracking and maintenance of the FSO links are also very easy and do not have any problem of frequent fiber faults due to digging during the setup of the new links and maintenance of the existing links etc. (Alkholidi and Altowij 2014). Hence, FSO is a very convenient technology to expand the legacy network within a short time to handle the sudden traffic growth of the users. However, FSO link connectivity is highly dependent on inconsistent weather conditions (Malik and Singh 2015). During rain and high haze, the FSO link losses may become significant and consequently, the receiver may not receive the minimum required power. Alternately, service providers need to transmit very high optical powers, which may not be desirable due to harmful laser effects to living beings. Also, as the energy resources are limited in nature, therefore, must have to be utilized optimally to use them in the long 
run. Moreover, large energy consumption will lead to the release of enormous environmental hazardous gasses that cannot be tolerated in smart and innovative cities. There must be some protocols to monitor and control the release of such gasses.

Pure SMF based latency-aware and energy-efficient hybrid WDM/TDM-PON is proposed in Garg et al. (2020) which has the direct optical distribution network (ODN) interconnection capability. Individually, both the SMF and the FSO have their merits and demerits and the integration of SMF and FSO seems attractive and one of the best solutions for providing high speed, energy-efficient, and reliable internet services. The existing network may be expanded with the FSO links very quickly. Recently, we have proposed a hybrid FSO/SMF link based WDM optical network architecture (Garg et al. 2021). However, this architecture was not capable of transmitting data at dual rates.

There are various architectures, which reported the integration of FSO with the SMF or WDM FSO implementation for leveraging the benefits of dual technology simultaneously. $32 \times 40$ Gbps WDM FSO link wad developed (Ciaramella et al. 2009) in which authors transmitted the signals at such high speed for the first time. They observed that the BER fluctuations for the entire day is very less and the WDM FSO could be the new possible technology to transmit high-speed data with good reliability and security. They further stabilized the signal power with incorporation of the saturated EDFA in the link and further stabilized the BER performance (Khazaali 2013). The performance of the WDM FSO link for 16 channels was analyzed for different type of optical filters including Gaussian, Bessel and Rectangular types (Aladeloba et al. June 2013). They achieved the good signal reception at $2.5 \mathrm{Gbps}$ for $2 \mathrm{~km}$ distance for low turbulence with a Q-factor of approximately 15. However, for moderate and high turbulences, the transmission distances was reduced to $1.7 \mathrm{~km}$ and $1.2 \mathrm{~km}$, respectively. To minimize the system cost and realizing the high bandwidth, high speed WDM FSO was demonstrated and found that the major causes for performance degradations of WDM FSO links were inter-channel interferences, EDFA ASE noise and scintillation. These could be managed by various advanced technologies (Elsayed and Yousif 2020). Analysis of WDM FSO link for different rain situations has been carried out in Shah and Kothari (2014). Authors transmitted data at $2.5 \mathrm{Gbps}$ and achieved the acceptable BER of $1 \times 10^{-9}$ for transmission distances of $3 \mathrm{~km}, 6.1 \mathrm{~km}$ and $15.6 \mathrm{~km}$ for high, medium and low rains. The study of numerous environmental parameters for bidirectional link for WDM FSO network has been done in Liaw et al. (2017). The authors transmitted data at a rate of $2 \times 4 \times 10 \mathrm{Gbps}$ for the distance of $25 \mathrm{~m}$. The observed power penalty for bidirectional signal is less than $0.8 \mathrm{~dB}$ and $0.2 \mathrm{~dB}$ as compared to back to back and unidirectional transmission. For fault protection at the distribution network, an interesting architecture is addressed (Hayle et al. 2021), which connected the disconnected ONUs through the neighboring ONU by creating the dynamic FSO link between the ONUs. However, each ONU was connected with the only one neighboring ONU and on failure of the adjoining ONU, the protection mechanism will not work. There might be fair chances of link failure between AWG output port and optical coupler. In such case, any of the ONU will not receive the signal and the purpose of architecture fails. $32 \times 5$ Gbps PON integrated with FSO protection was addressed in Mirza et al. (2020). The architecture enabled the protection of the commercial centers by duplicating the FSO link in parallel with the fiber link between the polarization splitter and optical switch at distribution network. Authors analyzed the BER performance at the receiver for fiber and FSO link for different polarization mode dispersion (PMD) turbulences respectively.

A WDM FSO linked with fiber network was presented in Yeh et al. (2019), in which the signal was transmitted for $25 \mathrm{~km}$ through the fiber and $5 \mathrm{~m}$ through the free space. Authors demonstrated the bidirectional transmission for four pairs of line terminals and 
optical wireless units through the FSO link by simultaneously transmitting the downstream and upstream WDM signals. Hence, the proposed architecture not only provided the dedicated connection, but, also reduced the Rayleigh Backscattering noise. A hybrid optical fiber/ FSO based architecture was proposed for the bidirectional transmission of the signal in the cross bridge communication in Yu et al. (2015). Fiber Bragg grating based sensor was incorporated in the bridge to monitor the structural deformations in the bridge. On occurrence of any deformations in the bridge, the link was switched from the fiber to the FSO for ensuring the reliable uninterrupted connectivity. They achieved the power penalty below $1 \mathrm{~dB}$ for fiber and FSO link at the BER of $1 \times 10^{-9}$. A star ring based TWDM PON network was proposed in Yeh et al. (2020), providing the protection in the distribution network by connecting each ONU through the fiber link as well as FSO link. A fiber link was deployed between the remote node to all the ONUs through the optical splitter. The FSO link was created among two neighboring ONUs and virtually connected in the ring. Another interesting architecture based on optical fiber and FSO integration was proposed in Yeh et al. (2020). The architecture connected central office and various optical wireless units (OWU) through the optical fiber in the ring topology. In case of the fiber fault in the ring, the signal can be transmitted by transmitting some wavelengths clockwise and some wavelengths anticlockwise. The signal reaching at the OWU can be transmitted wirelessly as earlier. However, on occurrence of two fiber faults in the ring, the scheme will not work.

The transmission modeling in both SMF and FSO links is explained, briefly, as follows.

The guiding characteristics of lightwave signals can be defined by the normalized frequency, or the V- number of the optical fiber

$$
\mathrm{V}=\mathrm{a}(\omega / \mathrm{c})\left(\mathrm{n}_{1}^{2}-\mathrm{n}_{2}^{2}\right)^{1 / 2}
$$

where $\mathrm{a}$ is the fiber core radius and $\omega$ is the angular frequency of the lightwave signal. $\mathrm{n}_{1}$, $\mathrm{n}_{2}$ are the refractive index of the core and cladding, respectively. For step index SMF, the $\mathrm{V}$-number is less than 2.405.

For the SMF, optical signal transmission can be defined by the standard nonlinear Schrödinger equation

$$
\frac{\partial A}{\partial z}+\frac{i \beta_{2}}{2} \frac{\partial^{2} A}{\partial t^{2}}=-\frac{\alpha}{2} A+i \gamma|A|^{2} A
$$

where $\alpha$ is the fiber attenuation loss, which is a function of z. $\beta_{2}$ represents the dispersion parameter and $\gamma$ is the fiber nonlinearity parameter. The term $\frac{i \beta_{2}}{2} \frac{\partial^{2} A}{\partial t^{2}}$ defines the chromatic dispersion, and the term $i \gamma|A|^{2} A$ indicates the phase shift due to the intensity variations (Agrawal 2013).

For the FSO link, the losses are due to the atmospheric and geometric losses. Atmospheric losses are mainly due to light absorption and scattering. Geometric losses are due to the divergent angle of the transmitter and receiver aperture. The link losses are given by $\mathrm{Vu}$ et al. (2013)

$$
a_{v}=\frac{A}{\pi\left(\frac{\theta L}{2}\right)^{2}} e^{-\beta_{l} L}
$$

where $\beta_{l}$, L, A and $\theta a$ re the extension coefficient, transmission distance, area of the transmitter and angle of divergence, respectively.

The environmental attenuation, as a function of visibility, $\mathrm{V}$, is given by 


$$
\sigma=\frac{3.91}{V}\left(\frac{\lambda}{550}\right)^{-q}
$$

where $\lambda$ and $\mathrm{q}$ are the operating wavelength and the size of the scattering particles.

The attenuation in $\mathrm{dB} / \mathrm{km}$ is defined by

$$
A_{e}=\frac{17}{V[k m]}\left(\frac{0.55}{\lambda[\mu m]}\right)^{q} \geq 0
$$

Apart from the attenuation losses, signal transmitted through the FSO also suffers atmospheric turbulences, which are considered based on the Gamma-Gamma distribution

$$
P\left(I_{a}\right)=\frac{2(\alpha \beta)^{\frac{(\alpha+\beta)}{2}}}{\gamma(\alpha) \gamma(\beta)} I_{a}^{\frac{(\alpha+\beta)}{2}-1} K_{\alpha-\beta}\left(2 \sqrt{\alpha \beta I_{a}}\right)
$$

where $K$ is the modified Bessel function of the second kind and

$$
\begin{aligned}
& \alpha=\exp \left[\frac{0.49 \sigma_{R}^{2}}{\left(1+1.11 \sigma_{R}^{\frac{12}{5}}\right)^{\frac{7}{6}}}\right]-1 \\
& \beta=\exp \left[\frac{0.51 \sigma_{R}^{2}}{\left(1+0.69 \sigma_{R}^{\frac{12}{5}}\right)^{\frac{5}{6}}}\right]-1
\end{aligned}
$$

where $\sigma_{R}^{2}$ is the Rytov variance and its value is given by $1.23 \mathrm{C}_{\mathrm{n}}{ }^{2} \mathrm{k}^{7 / 6} \mathrm{~L}^{11 / 6}$. The constant $\mathrm{C}_{\mathrm{n}}{ }^{2}$ is the refractive index structure coefficient, which has different values for different turbulences. $\mathrm{L}$ and $\mathrm{k}$ are the link length and the wave number, respectively. The value of $\mathrm{C}_{\mathrm{n}}{ }^{2}$ is $5 \times 10^{-\mathrm{x}} \mathrm{m}^{-2 / 3}$, where $\mathrm{x}$ is $-17,-15$ and -13 for weak, moderate, and strong turbulence, respectively (Badar and Jha 2017; Badar et al. 2018).

The subject of this paper is focusing on the system design of SMF/FSO link. So, we here include a summarized modeling for both links and for more details, one can turn to Refs. (Vu et al. 2013; Badar and Jha 2017; Badar et al. 2018).

In this paper, we have proposed a dual-rate SMF/FSO integrated, reliable optical network architecture that can support the transmission of data at a variable rate ranging from 1 to 20 Gbps through an FSO link and/or SMF link. Depending on the weather conditions and physical link availability in adverse geographical locations, a particular link can be utilized. Since the network does not have a homogenous traffic load throughout the clock, depending on the traffic load, the proposed network supports the transmission of narrowband data (1 Gbps) and broadband data $(10 \mathrm{Gbps})$ to utilize the resources optimally and further reduce the energy consumption. The proposed hybrid SMF/FSO architecture is capable of transmitting the signals at dual rate (1 Gbps and $10 \mathrm{Gbps})$ as per the traffic load in the network, i.e., the proposed architecture utilizes the available OLT resources efficiently to reduce the energy consumption and available bandwidth efficiently. To the best of our knowledge, earlier reported hybrid SMF/FSO architectures did not include 
important dual rate transmission capability. The proposed architecture is highly reliable against any one of the link (SMF or FSO) failures or transceiver failures and is discussed in detail in the coming sections. The performance of the proposed architecture is verified using the Optisystem 17.1 simulation tool. The rest of the paper is organized as follows. The proposed architecture is explained in Sect. 2. Section 3 describes the working and operation. The obtained results are displayed and discussed in Sect. 4. Section 5 is devoted to the main conclusions.

\section{Proposed architecture}

The schematic diagram of the proposed architecture is represented in Fig. 1. The optical line terminal (OLT) located at the central office (CO) consists of $2 \mathrm{~N}$ transceivers (TRx) for transmitting $2 \mathrm{~N}$ wavelengths to serve N ODNs. At the OLT, half of the transceivers are operated at a broadband $10 \mathrm{Gbps}$ and a half at a narrow band $1 \mathrm{Gbps}$. At the receiver side, each ODN will receive two wavelengths; one at $10 \mathrm{Gbps}$ and another at 1 Gbps. Depending on the traffic load on the ODN, a particular TRx may be utilized for transmitting the broadband or narrowband data and another may be switched off to reduce the energy consumption. The transmitted $2 \mathrm{~N}$ wavelengths are multiplexed and combined by the $2 \mathrm{~N} \times 1$ MUX. The OLT also has a wavelength division multiplexed (WDM) overlay transceiver which is used for broadcasting a common message to all the ODNs and the connected users. In our architecture, we have used CWDM for multiplexing/demultiplexing two channels which are $20 \mathrm{~nm}$ apart. Channel 1 of CWDM is further supporting 8 downstream/upstream DWDM wavelengths ( $0.8 \mathrm{~nm}$ spaced) which are multiplexed by the WDM Mux. Channel 2 is supporting only a single wavelength (20 $\mathrm{nm}$ apart from the first wavelength of the DWDM grid) on which WDM overlay wavelength is multiplexed/demultiplexed. In the proposed architecture, the CWDM is used at optical line terminal (OLT) and remote node (RN). OLT CWDM is multiplexing the downstream/upstream signals with the WDM overlay signal. RN CWDM is demultiplexing the WDM overlay signal from the stream of the multiplexed signals. The OLT CWDM is connected to the $1 \times 250 \mathrm{GHz}$ WDM interleaver, which distributes the even and odd multiple of $50 \mathrm{GHz}$ wavelengths to its two output ports. One output port of the

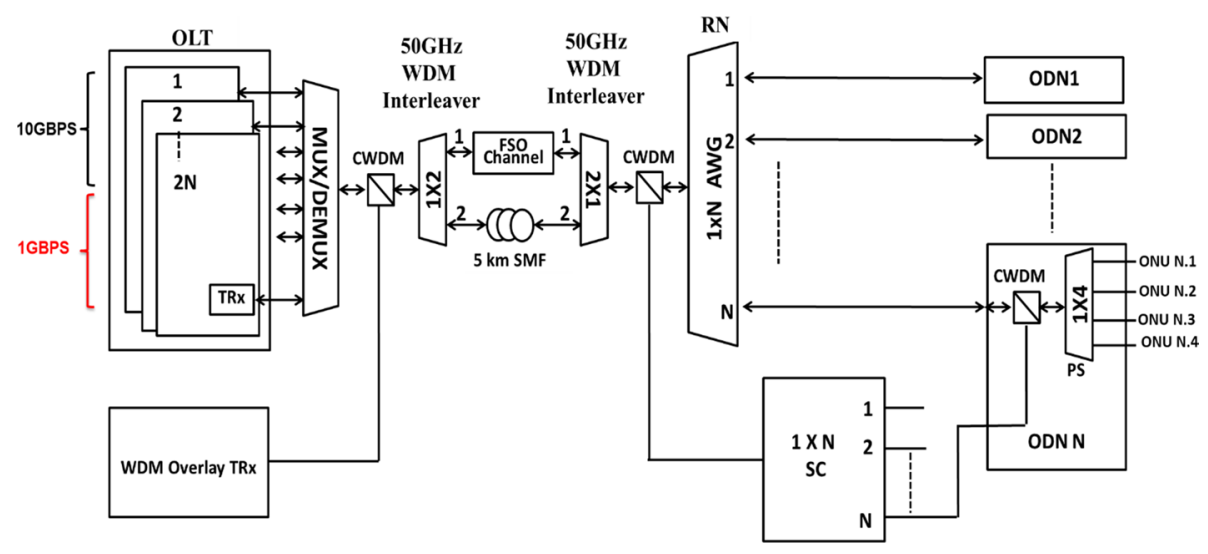

Fig. 1 Proposed dual-rate dual-link hybrid FSO/SMF architecture 
interleaver is connected to the FSO link and the other output port is connected to the SMF link for transmitting the data. At other end of the links, odd and even wavelength streams are combined by the $2 \times 150 \mathrm{GHz}$ WDM interleaver. The WDM overlay wavelength may be transmitted through any one of these two links by tuning the transmitted wavelength properly. At the remote node (RN), the WDM overlay wavelength is separated by CWDM and is diverted towards $1 \times \mathrm{N}$ star coupler (SC). $2 \mathrm{~N}$ downstream wavelengths are applied to the $1 \times \mathrm{N}$ arrayed waveguide grating (AWG) and the overlay wavelength is applied to the $1 \times \mathrm{N} \mathrm{SC}$. The AWG distributes the $2 \mathrm{~N}$ wavelengths to the $\mathrm{N}$ ODNs, i.e., each ODN is receiving two wavelengths for servicing the connected optical networking units (ONU). The SC broadcasts the overlay wavelength to all the ODNs and connected ONUs. The star coupler (SC) and power splitter (PS) are designed by fusing the multiple single mode fibers for the length of few millimeters. These devices work on the fact that the fraction of the transmitted signal in the single mode fiber gets spread into the cladding region and through evanescent power coupling process, signal power gets coupled in the other fiber. The fraction of the coupled power can be changed by changing the evanescent fields between the fibers. Furthermore the evanescent fields can be changed by changing the coupling length.

The proposed architecture can operate on the FSO link or SMF link depending on the weather conditions. If the weather is clear, the FSO link may be preferred as it has low losses and gives some unique advantages as discussed before. In rain or other adverse weather conditions, the SMF may be preferred rather than FSO as it has significantly low losses. The transmission of data from these two links depends on the wavelengths transmitted by the OLT. If the transmitted wavelengths are even multiple of $50 \mathrm{GHz}$, then, the interleaver pushes the incoming wavelengths to converge at the first output port and transmit through the FSO. If the transmitted wavelengths are an odd multiple of $50 \mathrm{GHz}$, then, the interleaver pushes the incoming wavelengths to converge at the second output port and transmit through the SMF. Hence, one can switch the link by tuning the transmitted wavelengths by $50 \mathrm{GHz}$.

The link switching from the FSO to SMF is simply depending on the transmitted wavelength from the optical line terminal (OLT) located at the central office. So, we need to tune the transmitted wavelength by $50 \mathrm{GHz}$ from the readily available tunable transmitters. The used $50 \mathrm{GHz}$ interleaver is working as a filter for separating the odd and even multiples of $50 \mathrm{GHz}$ wavelengths. All the even and odd multiples of $50 \mathrm{GHz}$ wavelengths would automatically be diverted on the FSO and SMF links respectively with the help of the $50 \mathrm{GHz}$ interleaver. Consequently, the link switching could be performed just by tuning the transmitted wavelength from the OLT without developing any specific complex protocol.

Concerning the data loss, in reality, one can transmit data through any link at any time, as both the links are always functional. Once the data is transmitted at a particular wavelength, the transmission will take place on a link for which data is intended, i.e., either on FSO or on SMF link. Link can be switched during transmission and there may be loss of only few bits due to the availability of ultrafast lasers that can be switched very rapidly. However, in case of the fault in any of the links, the part of the signal may be lost and signal needs to be retransmitted on the other link by tuning the transmitted signal by $50 \mathrm{GHz}$

Commercial versions of the used components are also available (https://www.optcore.net/product/ Accessed from 25 Mar 2021; https://www.corning.com/opcomm/oemsolutions/worldwide/en/products/micro-optics-products/wavelength-management.html Accessed from $20 \mathrm{Feb} 2021$; https://www.fs.com/c/wdm-optical-access-1 Accessed from $20 \mathrm{Feb} 2021$ ) and can be used for the practical realization of the proposed architecture. 


\section{Working and operation}

Just for ease of simulation setup, we have considered eight TRx at OLT and four ODNs at the user end. The first four TRx are modulated at 10 Gbps and the remaining four TRx are modulated at 1 Gbps and a particular one or both the TRx may be used for transmitting the data according to the traffic load. The line width of the CW laser is $10 \mathrm{MHz}$ and the initial optical power is $0 \mathrm{dBm}$ (i.e. $1 \mathrm{~mW}$ ). At the user side, each ODN consists of four ONUs. So, the network is supporting up to 16 users. We have opted the grid of frequencies based on the recommendation G.692, first ITU-T specification for the WDM. The both downlink and uplink follows the standard $100 \mathrm{GHz}$ wavelength spacing that corresponds to $(193.1+n \times 0.1) \mathrm{THz}$ and $(195.1+\mathrm{n} \times 0.1) \mathrm{THz}$ respectively. where $\mathrm{n}$ represents integer value with a frequency spacing of $100 \mathrm{GHz}$ for two consecutive values. For transmitting the data through the FSO link, eight wavelengths (even multiple of $50 \mathrm{GHz}) 193.1 \mathrm{THz}$, 193.2 THz...193.8 THz, are transmitted from the OLT as shown in Fig. 2. At the other end of the link $(\mathrm{RN})$, these wavelengths are applied to the $1 \times 8$ AWG. The center frequency $\left(\mathrm{f}_{\mathrm{c}}\right)$ and the frequency spacing $(\Delta \mathrm{f})$ between two successive output ports of AWG are kept as 193.1 THz and $100 \mathrm{GHz}$, respectively.

Due to the cyclic property of the AWG (Ciaramella et al. 2009), the wavelengths 193.1 $\mathrm{THz}, 193.2 \mathrm{THz}, 193.3 \mathrm{THz}$ and $193.4 \mathrm{THz}$ will reach at port1, port2, port3, and port4, respectively. Similarly, the wavelengths $193.5 \mathrm{THz}, 193.6 \mathrm{THz}, 193.7 \mathrm{THz}$, and $193.8 \mathrm{THz}$ will start repeating the port1, port2, port3, and port4, respectively. The first four wavelengths are modulated at $10 \mathrm{Gbps}$ (broad band) and the remaining four wavelengths are modulated at $1 \mathrm{Gbps}$ (narrow band). Therefore, each ODN is receiving a broadband as well as a narrowband wavelength and depending on the traffic load, any one or both the wavelengths may be transmitted. For broadcasting the signal through the FSO link, even multiple of $50 \mathrm{GHz}$ wavelength (e.g. $194.0 \mathrm{THz}$ ) is transmitted from a WDM overlay transceiver. The WDM overlay wavelength is combined by the CWDM at the central office and is then transmitted to the FSO link. At the RN side, this WDM overlay wavelength is separated via another CWDM from the downstream wavelengths and is applied to $1 \times 4 \mathrm{SC}$. The SC broadcasts the incoming wavelength to all ODNs connected to it. The ODN consists of an additional CWDM that combines the downstream wavelength and the WDM overlay

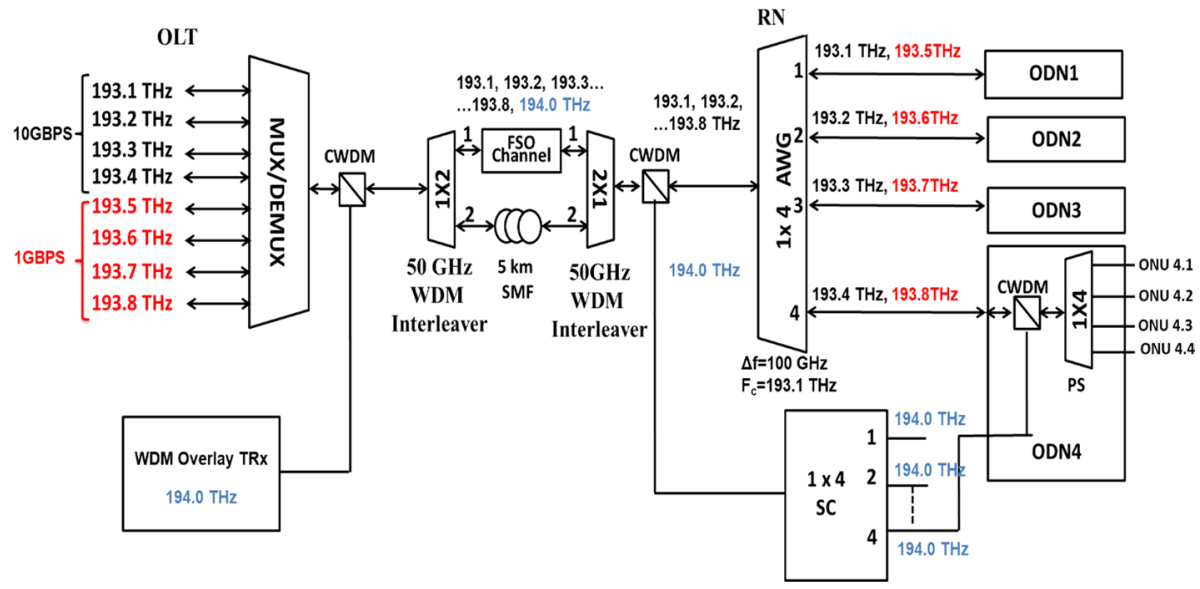

Fig. 2 Downstream and WDM overlay transmission through a FSO link 
wavelength. These wavelengths are further distributed to all four connected ONUs through an additional $1 \times 4 \mathrm{SC}$. Therefore, in the proposed architecture, a downstream wavelength is serving four ONUs and the broadcasted wavelength is serving 16 ONUs.

Figure 2 shows the path of the wavelengths which can be reached from the OLT to the ODNs. Each ODN is capable of receiving all the three wavelengths at any instant. However, a particular ONU cannot receive all the three wavelengths simultaneously. Therefore, few of the ONUs can be tuned at $10 \mathrm{Gbps}$, and few of the ONUs can be tuned at $1 \mathrm{Gbps}$ in order to utilize wavelength efficiently. Also, in case of failure of either $10 \mathrm{Gbps}$ TRx, the corresponding ONUs can be tuned at $1 \mathrm{Gbps}$ and vice versa. For broadcasting the common signal to all the ODN and associated ONUs, all the ONUs need to be tuned at WDM overlay wavelength.

Similar to the FSO link, the architecture can also transmit data through the SMF link just by tuning the transmitted wavelength by $50 \mathrm{GHz}$, i.e., by transmitting the odd multiple of $50 \mathrm{GHz}$ wavelengths $193.15 \mathrm{THz}, 193.25 \mathrm{THz}, 0.193 .85 \mathrm{THz}$ from OLT. Now, the center frequency of the RN AWG is changed to $193.15 \mathrm{THz}$. Therefore port1, port2, port3, and port 4 of AWG will receive the two wavelengths one broadband and another narrowband (193.15 THz, 193.55 THz), (193.25 THz, 193.65 THz), (193.35 THz, $193.75 \mathrm{THz})$ and $(193.45 \mathrm{THz}, 193.85 \mathrm{THz})$ respectively as shown in Fig. 3. These wavelengths can be used similarly as described for the FSO link. For broadcasting a signal through SMF, the WDM overlay transmits the $50 \mathrm{GHz}$ shifted wavelength $194.05 \mathrm{THz}$ (odd multiple of $50 \mathrm{GHz}$ ) so that interleaver diverts it at port2 rather port1. The RN CWDM diverts the WDM overlay wavelength towards the $1 \times 4 \mathrm{SC}$ and broadcasts to all the ODNs and the connected ONUs.

WDM overlay wavelength can be used for broadcasting the downstream data to all available users in the network. As depicted in Figs. 2 and 3, the overlay wavelength signal is broadcasted via the $1 \times 4$ PS. Also, in case of failure of any WDM TRx located at the OLT, the service will be interrupted at the receiver side. However, the service can be continued on the overlay wavelength until the recovery of the failed WDM TRx. The best criteria of the proposed architecture is that it is capable of providing dynamic wavelength allocation to the ODNs and its connected ONUs just by increasing the frequency spacing $\Delta \mathrm{f}$ of the RN AWG. Nowadays, with the ever-increasing demand for wavelength shift in DWDM

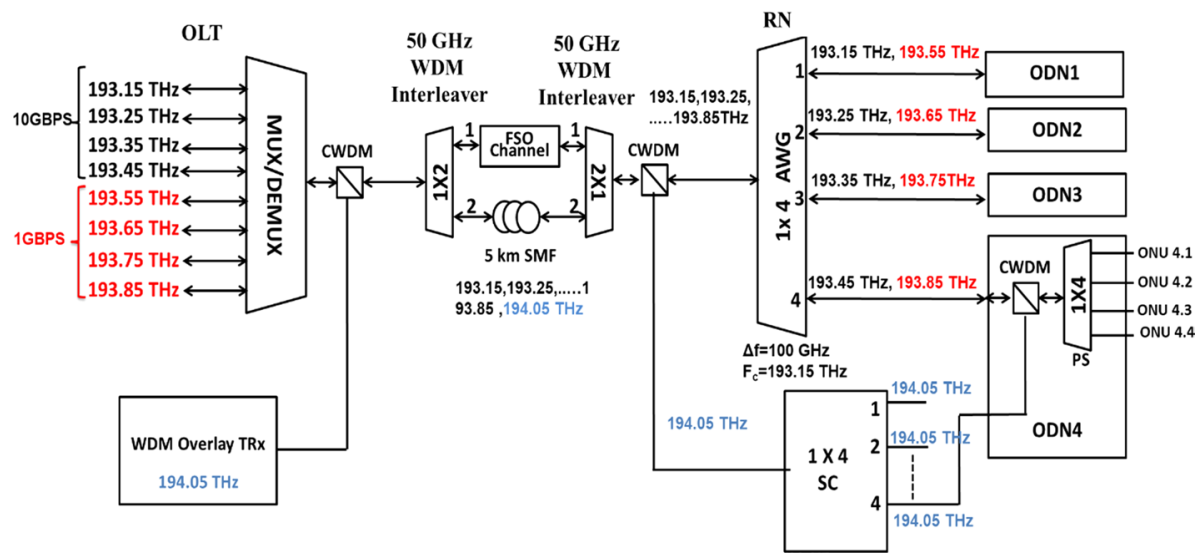

Fig. 3 Downstream and WDM overlay transmission through a SMF link 
technology, electro-optical tunable arrayed waveguide gratings (Horn et al. 2012) and thermos-optical tunable arrayed waveguide gratings (Toyoda et al. 2001; Leijtens et al. 2006) are readily available. The center frequency of AWG depends upon $n_{\text {eff }}$, given by $\lambda_{c}=2 n_{\text {eff }}$ $\cdot \Lambda$ (Horn et al. 2012). Just by changing $\mathrm{n}_{\text {eff }}$ by means of above-mentioned techniques, the central frequency can be easily changed. A wavelength shift of $0.12 \mathrm{~nm} /{ }^{\circ} \mathrm{C}$ can be easily achieved by changing the temperature in a range of 30 to $40{ }^{\circ} \mathrm{C}$, i.e., wavelength can be shifted $\sim 4$ to $5 \mathrm{~nm}$ (Leijtens et al. 2006). Just by doubling the frequency spacing $(200 \mathrm{GHz})$ and tuning its center frequency $\mathrm{f}_{\mathrm{c}}$ ranging from $193.1 \mathrm{THz}$ to $193.2 \mathrm{THz}, 193.3 \mathrm{THz}$ and 193.4 THz for different wavelength allocations, two contiguous wavelengths can be transmitted at a single port of the AWG. Depending on the particular value of $f_{c}$, two contiguous wavelengths will appear at port1, and then the next two wavelengths will be appeared at port 2 and so on as depicted in Fig. 4. For example, when $\mathrm{f}_{\mathrm{c}}$ is $193.1 \mathrm{THz}$ then each of ODN1, ODN2, ODN3 and ODN4 will receive the two wavelengths (193.1 THz, 193.2 THz), (193.3 THz, 193.4 THz), (193.5 THz, $193.6 \mathrm{THz})$ and (193.7 THz, $193.8 \mathrm{THz})$ respectively. So, ODN1, ODN2, ODN3 and ODN4 are modulated at $20 \mathrm{Gbps}, 20 \mathrm{Gbps}, 2$ Gbps, 2 Gbps respectively. When $\mathrm{f}_{\mathrm{c}}$ is changed to $193.2 \mathrm{THz}$, then ODN1, ODN2, ODN3 and ODN4 will receive the wavelengths $(193.2 \mathrm{THz}, 193.3 \mathrm{THz}),(193.4 \mathrm{THz}, 193.5 \mathrm{THz})$, $(193.6 \mathrm{THz}, 193.7 \mathrm{THz})$ and $(193.8 \mathrm{THz}, 193.1 \mathrm{THz})$ modulated at $20 \mathrm{Gbps}, 11 \mathrm{Gbps}, 2$ Gbps and $11 \mathrm{Gbps}$ respectively as shown in Fig. 4. A similar allocation will continue for the center frequencies $193.3 \mathrm{THz}$ and $193.4 \mathrm{THz}$.

Similar to the FSO link, the proposed architecture is capable of providing dynamic wavelength allocation for the SMF link as illustrated in Fig. 5. In this case, $\Delta \mathrm{f}$ of AWG is kept the same as $200 \mathrm{GHz}$ and $\mathrm{f}_{\mathrm{c}}$ is tuned at any one of the wavelengths $193.15 \mathrm{THz}$, 193.25 THz, $193.35 \mathrm{THz}$ or $193.45 \mathrm{THz}$, depending on the desired wavelength allocation. For example, if fc is $193.45 \mathrm{THz}$, then, ODN1, ODN2, ODN3 and ODN4 will receive the wavelengths (193.45 THz, 193.55 THz), (193.65 THz, 193.75 THz), (193.85 THz, 193.15 $\mathrm{THz}$ ) and (193.25 THz, $193.35 \mathrm{THz})$ modulated at $11 \mathrm{Gbps}, 2$ Gbps, $11 \mathrm{Gbps}$ and $20 \mathrm{Gbps}$, respectively.

Apart from the transmission through FSO or SMF link individually, the proposed architecture also supports the transmission through both links simultaneously. For this, some
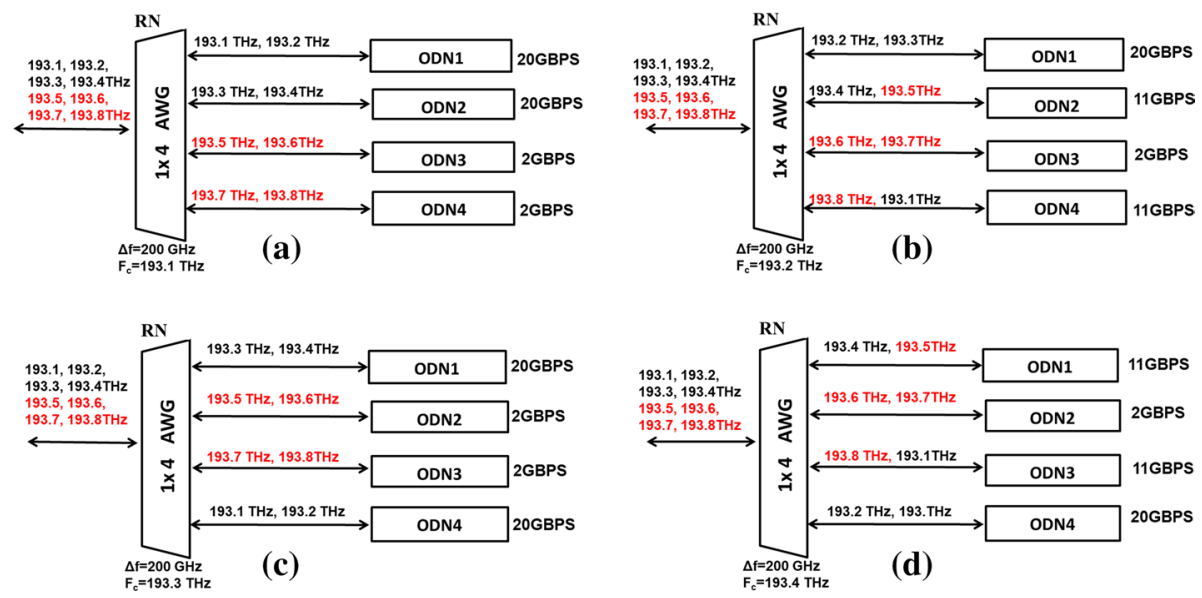

Fig. 4 Dynamic wavelength allocation to different ODNs for FSO communication $\left(\mathbf{a}: \mathrm{f}_{\mathrm{c}}=193.1 \mathrm{THz}\right.$, b: $\mathrm{f}_{\mathrm{c}}=193.2 \mathrm{THz}, \mathbf{c}: \mathrm{f}_{\mathrm{c}}=193.3 \mathrm{THz}$, and $\left.\mathbf{d}: \mathrm{f}_{\mathrm{c}}=193.4 \mathrm{THz}\right)$ 

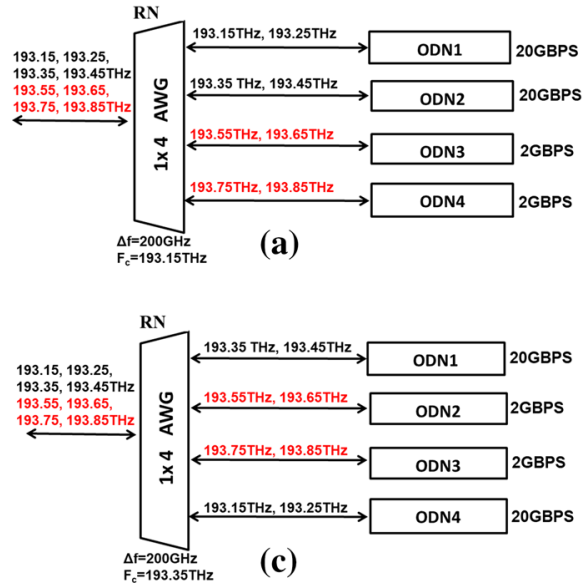
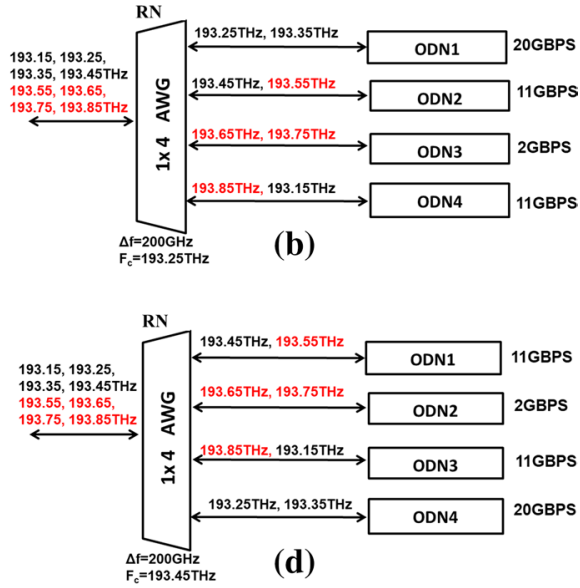

Fig. 5 Dynamic wavelength allocation to different ODNs for SMF communication $\left(\mathbf{a}: \mathrm{f}_{\mathrm{c}}=193.15 \mathrm{THz}, \mathbf{b}\right.$ : $\mathrm{f}_{\mathrm{c}}=193.25 \mathrm{THz}, \mathbf{c}: \mathrm{f}_{\mathrm{c}}=193.35 \mathrm{THz}$, and $\left.\mathbf{d}: \mathrm{f}_{\mathrm{c}}=193.45 \mathrm{THz}\right)$

wavelengths are transmitted at even multiple of $50 \mathrm{GHz}$ and some wavelengths are transmitted at odd multiple of $50 \mathrm{GHz}$ wavelengths. The even and odd multiple wavelengths will reach at port 1 and port 2 of $1 \times 2$ interleaver, respectively, i.e., even wavelengths will travel through the FSO link and odd wavelengths will travel through the SMF link. In case of a very heavy traffic load, both the links could be used simultaneously to avoid the overburden on a single link. The WDM overlay wavelength could also be transmitted through either FSO link or SMF link with downstream wavelengths simultaneously as given in Fig. $6 a$ and $b$.

FSO link may not work satisfactorily due to various weather conditions such as rain, fog, haze, etc. The SMF link may also fail due to digging or any other fiber faults. In such scenarios, the downstream data or WDM overlay data could be transmitted on the free link to gain reliability against the working link failure. However, for this reason, we have considered that both SMF and FSO link will not fail simultaneously, i.e., reliability can only be gained on failure of any one of the link only.

The proposed architecture also works well in upstream direction through both FSO and SMF links. The point to point upstream transmission for FSO and SMF links is depicted in Figs. 7a and b, respectively. For transmitting data through FSO link, eight upstream signals $(195.1,195.2,195.3,195.4 \mathrm{THz})$ at $10 \mathrm{Gbps}$ and $(195.5,195.6,195.7,195.8 \mathrm{THz})$ at $10 \mathrm{Gbps}$ are transmitted from the receiver side. These signals are applied to the $1 \times 4$ AWG having the center frequency and frequency spacing between two consecutive ports at $195.1 \mathrm{THz}$ and $100 \mathrm{GHz}$, respectively. Due to the cyclic property, these wavelengths are multiplexed and reach to the RN CWDM. As all the frequencies are even multiple of $50 \mathrm{GHz}$, these wavelengths are transmitted through the FSO link in the upstream direction as shown in Fig. 7a and reach to the OLT. For transmitting the signal through the SMF links as depicted in Fig. 7b, the upstream wavelengths are shifted by $50 \mathrm{GHz}$. The center frequency of AWG is also shifted by $50 \mathrm{GHz}$ and becomes $195.15 \mathrm{GHz}$. As the transmitted wavelengths are now odd multiple of $50 \mathrm{GHz}$, the WDM interleaver diverts all the wavelengths on the SMF link and reaches to the OLT. At OLT for both the cases including FSO and SMF, each receiver may receive two wavelengths transmitted at $10 \mathrm{Gbps}$ and $1 \mathrm{Gbps}$ and a particular wavelength can be used as per the traffic load. 


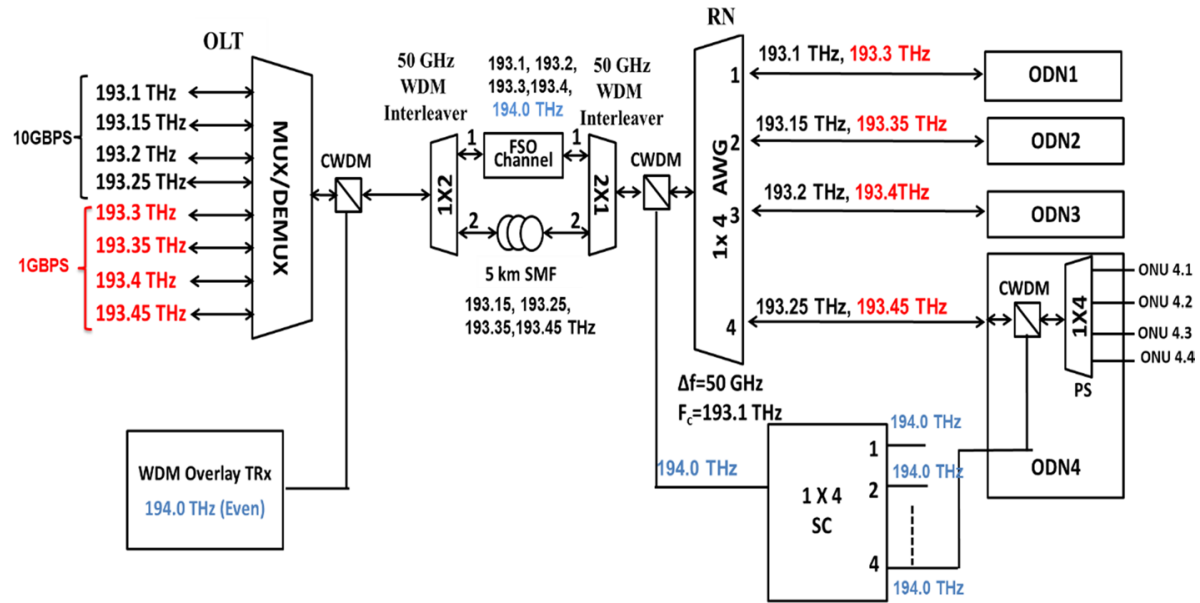

(a) Transmission of downstream data through both FSO/SMF with simultaneous FSO broadcasting.

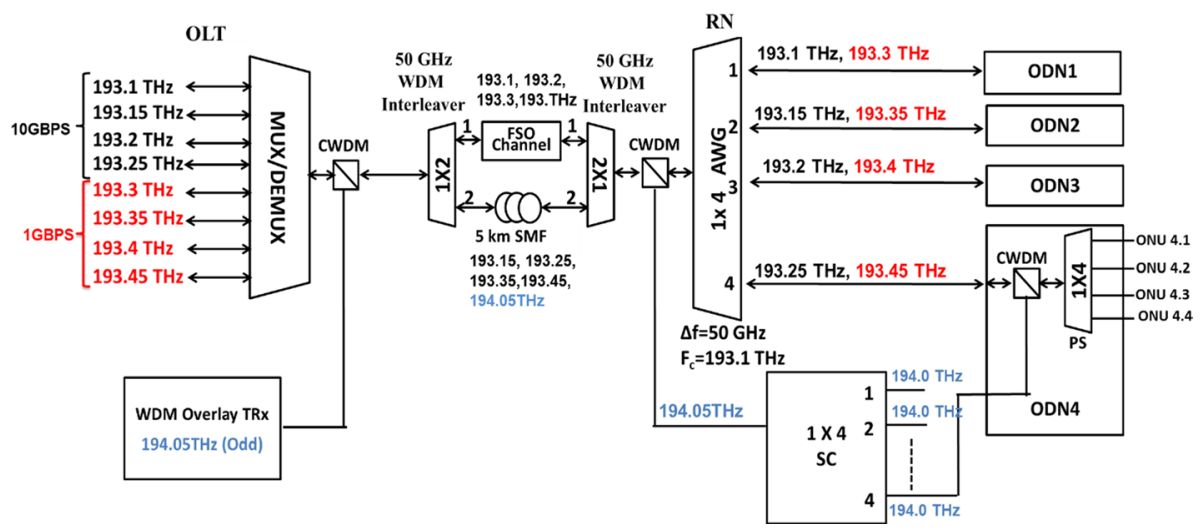

(b) Transmission of downstream data through both FSO/SMF with simultaneous SMF broadcasting.

Fig. 6 a Transmission of downstream data through both FSO/SMF with simultaneous FSO broadcasting. b Transmission of downstream data through both FSO/SMF with simultaneous SMF broadcasting

\section{Results and discussion}

The performance of the proposed architecture is verified with the help of the simulation tool Optisystem 17.1. The insertion loss for various components used in the link are considered as given in Table 1.

For clear weather conditions, the insertion loss for the whole FSO link and SMF links are almost the same and are about $20.5 \mathrm{~dB}$ for downstream and $17.5 \mathrm{~dB}$ for WDM overlay transmission, for $5 \mathrm{~km}$ link. However, in adverse weather conditions such as rain, the FSO link loss may become substantial, even up to $19.28 \mathrm{~dB} / \mathrm{km}$, and may not able to transmit data within an acceptable BER range or needs very high-transmitted 


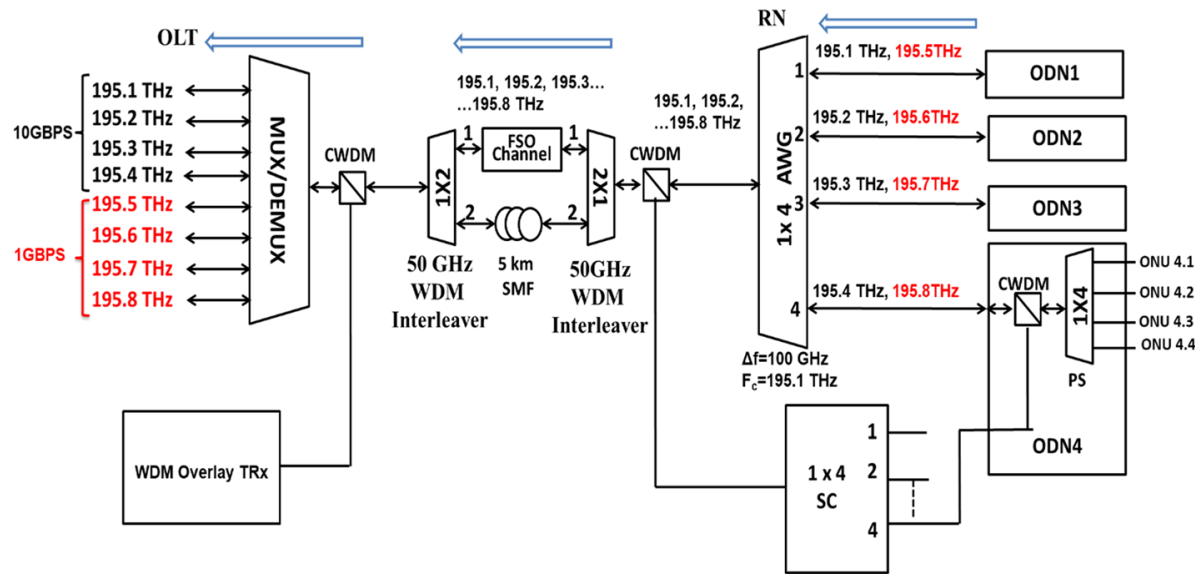

(a) Point to point FSO communication in upstream direction.

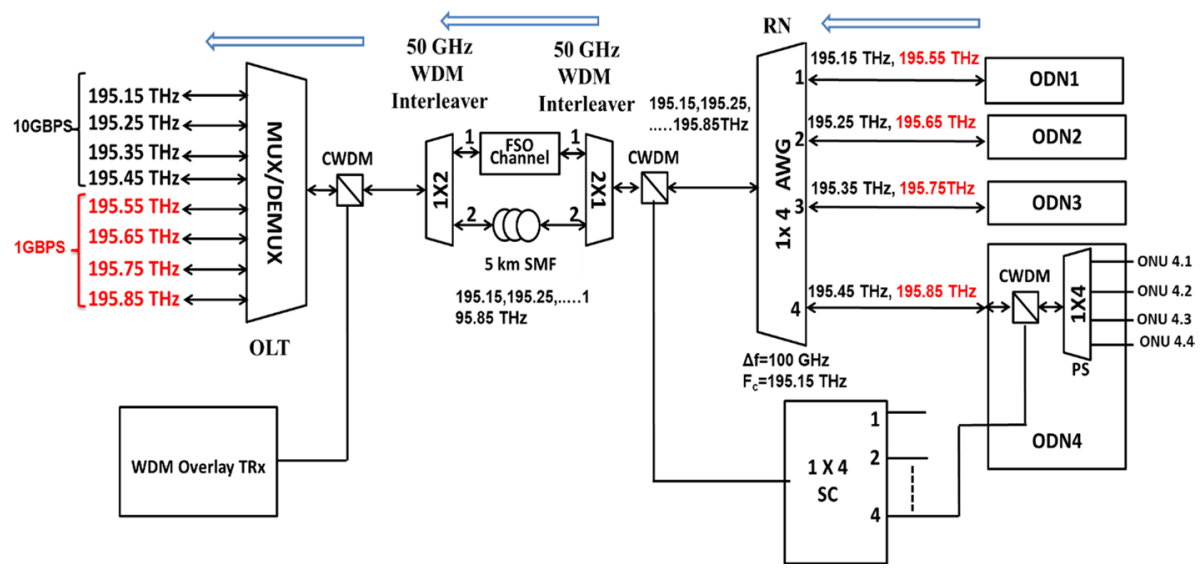

(b) Point to point SMF communication in upstream direction.

Fig. 7 a Point to point FSO communication in upstream direction. b Point to point SMF communication in upstream direction

powers (up to $500 \mathrm{~kW}$ ). It may need numerous repeaters and amplifiers in the link, which makes the system more complex, costly, and highly unstable. The addition of repeaters and amplifiers also decreases the reliability and speed of the system. Therefore, in such cases, an alternative link, SMF, may be preferred for better transmission reliability and link performance, which requires transmitted powers only in the range of a few $100 \mathrm{~mW}$.

The frequency-domain spectrum of the transmitted signals at OLT and received signals at four different ODNs are displayed in Fig. 8. At OLT, a total of eight wavelength signals are transmitted, out of which, four signals are modulated at 10 Gbps (broadband), and the remaining four are modulated at $1 \mathrm{Gbps}$ (narrowband). Each ODN is receiving two wavelengths; one modulated at $10 \mathrm{Gbps}$ and another is modulated at 1 Gbps. Any one or both the dedicated wavelengths could be transmitted to serve the particular ODN depending on the traffic load of that ODN. If the traffic load of the ODN 
Table 1 Link parameters used in simulation

\begin{tabular}{|c|c|}
\hline Components & Parameter \\
\hline Laser source & Linewidth $=10 \mathrm{MHz}$ and Initial power $=0 \mathrm{dBm}$ \\
\hline $8 \times 1 \mathrm{MUX} / \mathrm{DeMUX}$ & Loss $=5 \mathrm{~dB}$ \\
\hline CWDM & Loss $=0.5 \mathrm{~dB}$ \\
\hline $1 \times 2$ Interleaver & Loss $=0.5 \mathrm{~dB}$ \\
\hline FSO & $\begin{array}{l}\text { Loss }=0.233-19.28 \mathrm{~dB} / \mathrm{km} \text { depending on a } \\
\text { particular weather condition }\end{array}$ \\
\hline SMF & $\begin{array}{l}\text { Attenuation loss }=0.2 \mathrm{~dB} / \mathrm{km} \\
\text { Dispersion }=16.75 \mathrm{ps} / \mathrm{nm} / \mathrm{km} \\
\text { Dispersion slope }=0.075 \mathrm{ps} / \mathrm{nm}^{2} / \mathrm{km} \\
\text { Nonlinear index coefficient }=2.6 \times 10^{-20} \mathrm{~m}^{2} / \mathrm{W}\end{array}$ \\
\hline EDFA & Noise Figure $=4 \mathrm{~dB}$ \\
\hline $1 \times 4 \mathrm{SC} / 1 \times 4 \mathrm{PS}$ & $\begin{array}{l}\text { Insertion loss }=7 \mathrm{~dB}, \\
\text { Coupling coefficient }=0.5\end{array}$ \\
\hline $1 \times 4$ AWG & $\begin{array}{l}\text { Insertion loss }=5 \mathrm{~dB}, \\
\text { Center frequency }=193.1 \mathrm{THz}, \\
\text { Bandwidth }=10 \mathrm{GHz}, \\
\text { Frequency spacing }=100 \mathrm{GHz} \text {, } \\
\text { Noise threshold }=-100 \mathrm{~dB}\end{array}$ \\
\hline
\end{tabular}

Higher values of EDFA noise figure will increase the phase noise causing a degradation in the system performance
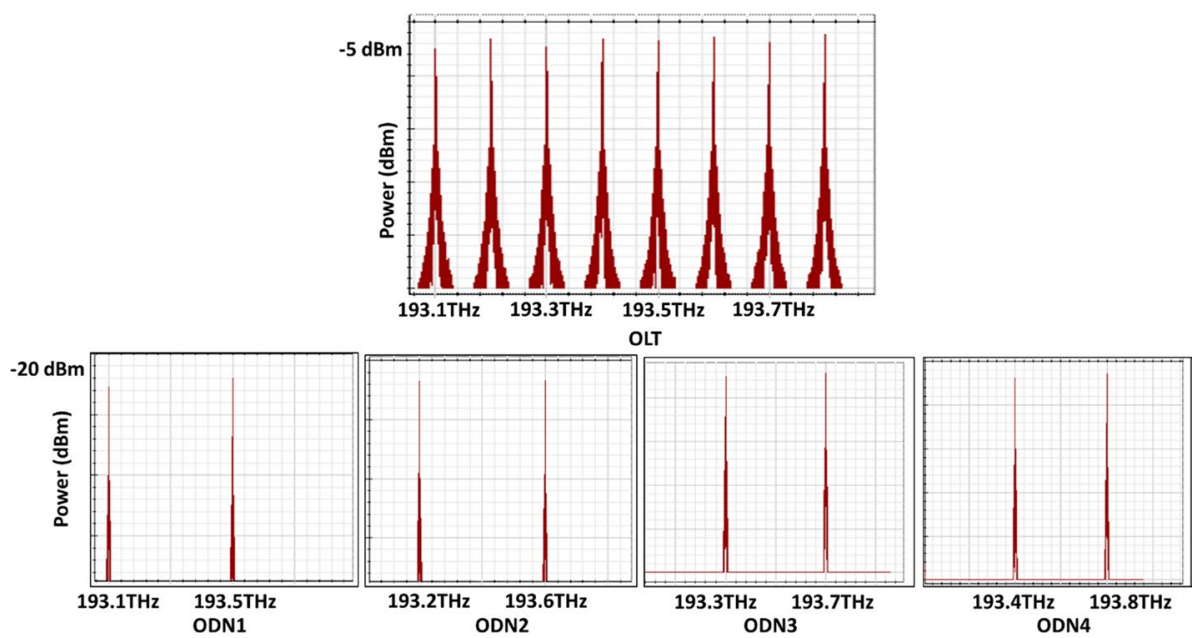

Fig. 8 Spectrum of transmitted and received signals at OLT and ODN(s)

is below a certain threshold, then, only one wavelength is transmitted and the TRx corresponding to the other wavelength is switched off to reduce the energy consumption.

The time-domain representation of the transmitted signal at OLT and the received signal at the ODN1 for both FSO and SMF links are shown in Fig. 9. The received signals indicate the clear and proper reception of the transmitted signals at both 1 Gbps and $10 \mathrm{Gbps}$ for both links. The SMF link has slightly higher signal distortion due to the various nonlinear effects occurring in it. However, the received signal quality is within 

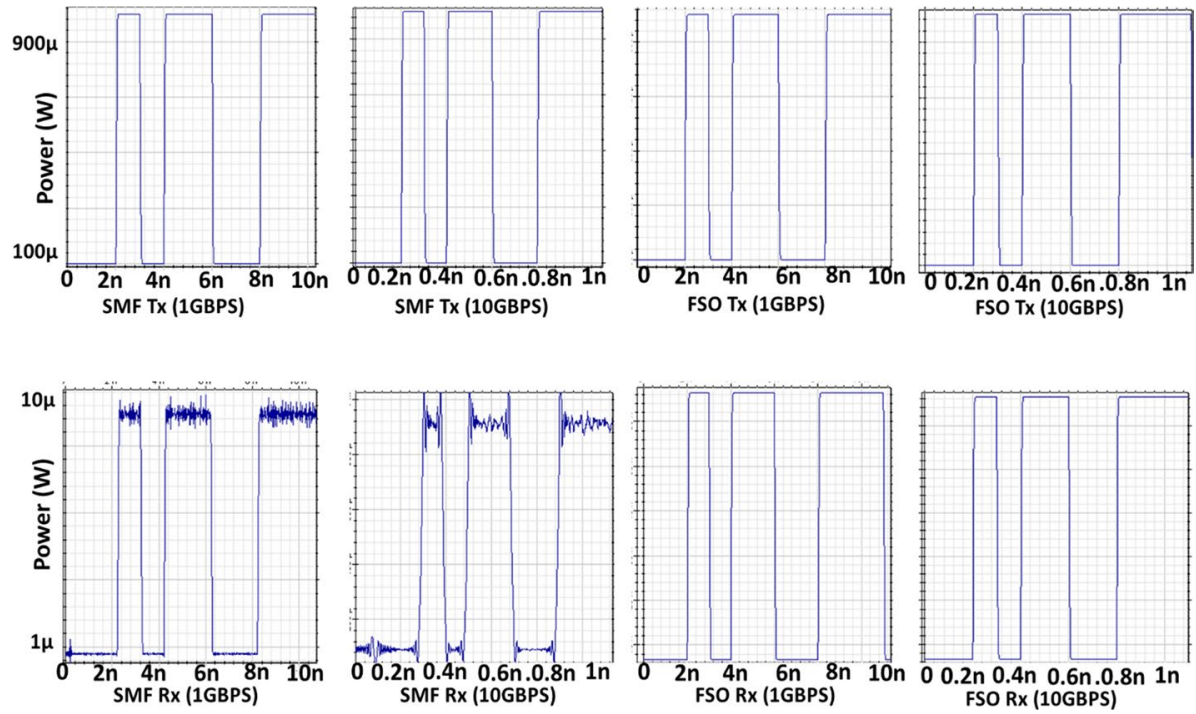

Fig. 9 Time-domain representation of transmitted and received signals for FSO and SMF

the acceptable quality of service (QoS) limit and can easily be detected with the simple detector.

The BER performance of the proposed architecture at $10 \mathrm{Gbps}$ and $1 \mathrm{Gbps}$ for both FSO and SMF links are illustrated in Fig. 10, which indicates the perfect reception of transmitted signals within the acceptable BER range. So, the proposed architecture is well suited for providing a reasonably better QoS. For BER performance of $1 \times 10^{-9}$ and quality factor above 6, the minimum required optical powers for $1 \mathrm{Gbps}$ FSO and 1 Gbps SMF links are $-33.7 \mathrm{dBm},-33 \mathrm{dBm}$, respectively. There is a link power penalty of $0.7 \mathrm{~dB}$ for SMF as compared to FSO due to its various nonlinear effects. For $10 \mathrm{Gbps}$, the minimum required optical powers for FSO and SMF links are $-28.5 \mathrm{dBm},-27.5 \mathrm{dBm}$, respectively, i.e., at 10 Gbps, the SMF link power penalty increases to approximately $1 \mathrm{~dB}$.

The link losses for the FSO channel are highly dependent on environment. For rainy and haze weather conditions, the FSO channel losses may become very high and restrict

Fig. 10 BER performance for FSO and SMF link for narrowband and broadband transmission

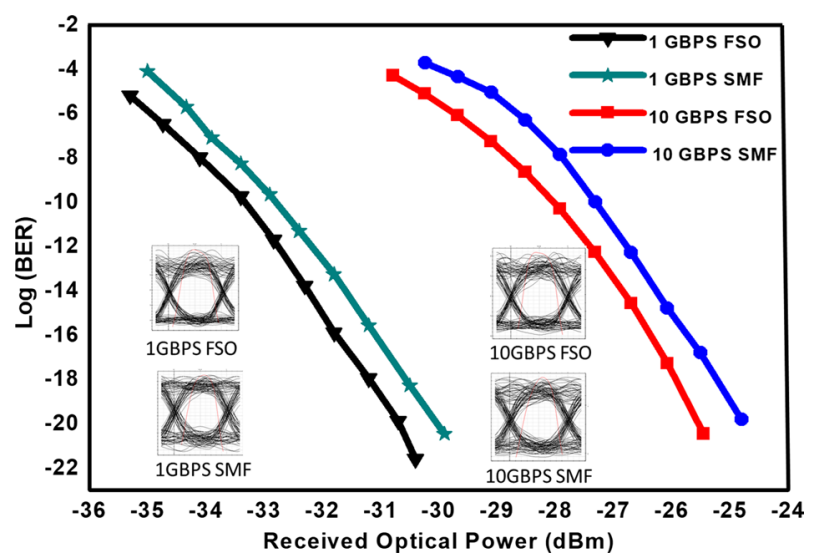


the power budget fulfillment. In such cases, FSO links may require very high-transmitted powers as compared to the SMF link. Due to the limitations of very high-transmitted powers in FSO, SMF links may be preferred at the cost of very small nonlinear effects and power.

Corresponding eye diagrams are also depicted in the insets that indicate the wide eye opening and no overlapping of the different bits. Figure 9 is plotted for all weather conditions. However, for clear weather condition, the data is transmitted on the FSO link and for other weather conditions such as rain, haze, etc., the data is transmitted on the SMF so that the performance is not deteriorated.

The proposed hybrid architecture is more reliable as compared to pure FSO or pure SMF link against any OLT TRx failure or link failure. The reliability matrix of the proposed architecture is given in Table 2.

At OLT, half of the TRx is modulated at $10 \mathrm{Gbps}$ and the remaining half is modulated at 1 Gbps. Depending on the traffic load on the particular ODN, any one or both the TRx are used for transmitting the wavelengths. If the load is less than $1 \mathrm{Gbps}$, then, $10 \mathrm{Gbps}$ TRx (corresponding to $10 \mathrm{Gbps}$ module) is switched OFF and the 1 Gbps TRx (corresponding to 1 Gbps module) is switched $\mathrm{ON}$ to reduce the energy consumption. If traffic load on the particular ODN is greater than 1 Gbps and less than $10 \mathrm{Gbps}$, then the 10 Gbps TRx is switched ON and the 1 Gbps TRx is switched OFF. If traffic load further increases and is greater than $10 \mathrm{Gbps}$, then, both the TRX are switched ON. However, the architecture is only capable to provide services up to $11 \mathrm{Gbps}$ only.

For dedicated services, normally, the WDM overlay TRx is switched OFF. For broadcasting the signal to all ODNs, it is switched ON. However, the WDM overlay TRx can also provide the services in case of any emergency or failure in both the dedicated TRx, i.e., each ODN can be serviced by three TRx as explained in the following paragraph.

Up to $1 \mathrm{Gbps}$ traffic load at the ODN, in case of the $1 \mathrm{Gbps}$ TRx failure, the ODN can be serviced by 10 Gbps TRx or WDM overlay TRx. Therefore, up to 1 Gbps traffic load, the proposed architecture is $200 \%$ more reliable against the failure of the OLT TRx. For traffic load greater than $1 \mathrm{Gbps}$ and less than $10 \mathrm{Gbps}$, and in case of failure of 10 Gbps TRx, the ODN can be serviced by any of the 1 Gbps or WDM overlay TRx without any service disruption. In this case, the proposed architecture is also $200 \%$ more reliable against TRx failure. However, as the ODN is having a traffic load greater than $1 \mathrm{Gbps}$, there may be some compromise with the transmission speed or QoS until the recovery of the failed TRx, but, one can continue the services without any service disruption. In case of traffic load greater than $10 \mathrm{Gbps}$ and limited up to $11 \mathrm{Gbps}$, both the $1 \mathrm{Gbps}$ TRx and 10 Gbps TRx are required, and in case of failure of any one of the TRx, the services may be continued on the WDM overlay TRx only. Therefore, for this case, the architecture is $100 \%$ more reliable.

In all the above three cases, the data could be transmitted on either the FSO link or the SMF link depending on the weather conditions. Normally, any of the links can be used efficiently and its architecture is $100 \%$ more reliable against link failure. However, in adverse weather conditions, only the SMF link is preferred and on its failure, more power needs to be transmitted for the FSO link to compensate for the link losses and maintain the transmission reliability. Therefore, depending on the priority, network service providers can choose either transmitted power or service reliability.

To check the energy-saving capability of the proposed dual-rate, dual-link FSO/SMF architecture, we have considered the arbitrary environmental conditions for a month, and they are given in Table 3. The FSO link loss for various weather conditions is given in Table 4 (Alkholidi and Altowij 2014). We have also considered that each day, out of $24 \mathrm{~h}$, 


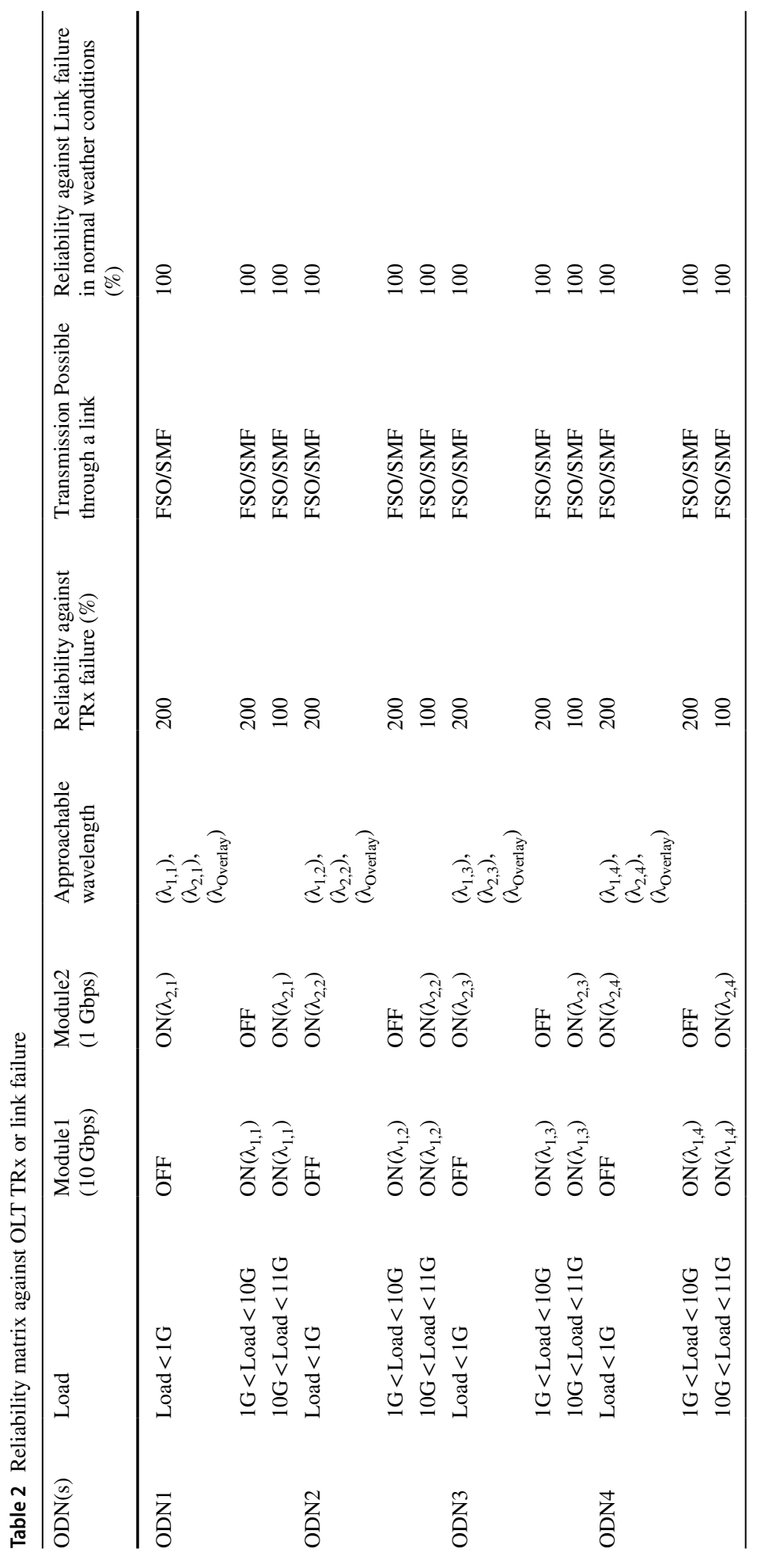


Table 3 Arbitrary environmental conditions for 4 weeks

\begin{tabular}{lllll}
\hline Day/Week & Week1 & Week2 & Week3 & Week4 \\
\hline Monday & Clear & Low Rain & Clear & Moderate Rain \\
Tuesday & High Haze & Moderate Rain & Clear & Low Rain \\
Wednesday & Low Haze & Low Rain & Low Haze & Low Rain \\
Thursday & Low Rain & Low Rain & High Haze & Low Rain \\
Friday & Moderate Rain & Low Rain & High Haze & Low Rain \\
Saturday & Low Rain & Clear & Low Haze & Clear \\
Sunday & Low Rain & Clear & Moderate Rain & Low Haze \\
\hline
\end{tabular}

"FSO" word is highlighted in bold just to differentiate it from the "SMF" for better readability

Table 4 FSO link loss for various weather conditions

\begin{tabular}{ll}
\hline Weather situation & $\begin{array}{l}\text { Losses }(\mathrm{dB} / \mathrm{km}) \\
(\text { Alkholidi and Altowij } \\
2014)\end{array}$ \\
\hline Clear & 0.233 \\
Low Haze & 0.55 \\
High Haze & 2.37 \\
Low Rain & 6.27 \\
Moderate Rain & 9.64 \\
High Rain & 19.28 \\
\hline
\end{tabular}

\begin{tabular}{lllll}
\hline Day/Week & Week1 & Week2 & Week3 & Week4 \\
\hline Monday & FSO & SMF & FSO & SMF \\
Tuesday & SMF & SMF & FSO & SMF \\
Wednesday & SMF & SMF & SMF & SMF \\
Thursday & SMF & SMF & SMF & SMF \\
Friday & SMF & SMF & SMF & SMF \\
Saturday & SMF & FSO & SMF & FSO \\
Sunday & SMF & FSO & SMF & SMF \\
\hline
\end{tabular}

"FSO" word is highlighted in bold just to differentiate it from the "SMF" for better readability
Table 5 Preferred links on different days if link loss $<0.25 \mathrm{~dB} / \mathrm{km}$ each ODN has the representative traffic load $<1 \mathrm{Gbps}, 1 \mathrm{Gbps}<\operatorname{load}<10 \mathrm{Gbps}$, and 10 Gbps $<$ load $<11$ Gbps for $7.2 \mathrm{~h}, 12 \mathrm{~h}$ and $4.8 \mathrm{~h}$ respectively.

Depending on the particular weather condition, the network service providers can select either the FSO link or the SMF link. For some cases, the FSO link is preferred only when the weather is quite clear and link loss is within $0.25 \mathrm{~dB} / \mathrm{km}$. For some instances, the FSO link may be utilized even if the link losses are up to $1 \mathrm{~dB} / \mathrm{km}$ or $2.5 \mathrm{~dB} / \mathrm{km}$. Depending on the link losses, three different cases $(<0.25 \mathrm{~dB} / \mathrm{km},<1 \mathrm{~dB} / \mathrm{km}$, and $<2.5 \mathrm{~dB} / \mathrm{km})$ are 
Table 6 Preferred links on different days if link loss $<1 \mathrm{~dB} /$ $\mathrm{km}$

\begin{tabular}{lllll}
\hline Day/Week & Week1 & Week2 & Week3 & Week4 \\
\hline Monday & FSO & SMF & FSO & SMF \\
Tuesday & SMF & SMF & FSO & SMF \\
Wednesday & FSO & SMF & FSO & SMF \\
Thursday & SMF & SMF & SMF & SMF \\
Friday & SMF & SMF & SMF & SMF \\
Saturday & SMF & FSO & FSO & FSO \\
Sunday & SMF & FSO & SMF & FSO \\
\hline
\end{tabular}

"FSO" word is highlighted in bold just to differentiate it from the "SMF" for better readability

Table 7 Preferred links on different days if link losses $<2.5 \mathrm{~dB} / \mathrm{km}$

\begin{tabular}{lllll}
\hline Day/Week & Week1 & Week2 & Week3 & Week4 \\
\hline Monday & FSO & SMF & FSO & SMF \\
Tuesday & FSO & SMF & FSO & SMF \\
Wednesday & FSO & SMF & FSO & SMF \\
Thursday & SMF & SMF & FSO & SMF \\
Friday & SMF & SMF & FSO & SMF \\
Saturday & SMF & FSO & FSO & FSO \\
Sunday & SMF & FSO & SMF & FSO \\
\hline
\end{tabular}

"FSO" word is highlighted in bold just to differentiate it from the "SMF" for better readability

considered and the preferred links on different days of a month are given in Tables 5, 6, and 7 , respectively.

By considering the environmental conditions of a month given in Table 3 , and assuming each ODN has the representative traffic load $<1 \mathrm{Gbps}, 1 \mathrm{Gbps}<\operatorname{load}<10 \mathrm{Gbps}$, and 10 Gbps $<$ load $<11$ Gbps for 7.2 h, $12 \mathrm{~h}$ and $4.8 \mathrm{~h}$, respectively. Energy-saving of the proposed architecture w.r.t the pure FSO link for different weeks are given by the following equations:

$$
\begin{aligned}
& E_{\text {Saving }}(\text { Week })=\sum_{d=1}^{7}\left[\begin{array}{l}
\left\{E_{10 G}(F S O)_{d}+E_{1 G}(F S O)_{d}\right\} \text { PureFSO } \\
-\left\{E_{10 G}(F S O)_{d}+E_{1 G}(F S O)_{d}+E_{10 G}(S M F)_{d}+E_{1 G}(S M F)_{d}\right\} \text { Hybrid }
\end{array}\right] \\
& \quad E_{\text {Saving }(\text { Week })} \\
& =\sum_{d=1}^{7}\left\{P_{10 G}(F S O)_{d} \cdot h_{10 G}(F S O)_{d}+P_{1 G}(F S O)_{d} \cdot h_{1 G}(F S O)_{d}\right\} \text { PureFSO } \\
& \quad-\left\{P_{10 G}(F S O)_{d} \cdot h_{10 G}(F S O)_{d}+P_{1 G}(F S O)_{d} \cdot h_{1 G}(F S O)_{d}+P_{10 G}(S M F)_{d} \cdot h_{10 G}(S M F)_{d}+P_{10 G}(S M F)_{d} \cdot h_{10 G}(S M F)_{d}\right\} \text { Hybrid }
\end{aligned}
$$




$$
\begin{gathered}
E_{\text {Saving }}(\%)=1-\frac{\sum_{d=1}^{7}\left\{E_{10 G}(F S O)_{d}+E_{1 G}(F S O)_{d}+E_{10 G}(S M F)_{d}+E_{1 G}(S M F)_{d}\right\} \text { Hybrid }}{\sum_{d=1}^{7}\left\{\sum_{d=1}^{7} E_{10 G}(F S O)_{d}+E_{1 G}(F S O)_{d}\right\} \text { PureFSO }} \\
E_{\text {Saving }}(\%)=1-\frac{\sum_{d=1}^{7}\left\{P_{10 G}(F S O)_{d} \cdot h_{10 G}(F M F)_{d} \cdot h_{10 G}(S M F)_{d}+P_{1 G}(S M F)_{d} \cdot h_{1 G}(S M F)_{d}\right\} \text { Hybrid }}{\sum_{d=1}^{7}\left\{P_{10 G}(F S O)_{d} \cdot h_{10 G}(F S O)_{d}+P_{1 G}(F S O)_{d} \cdot h_{1 G}(F S O)_{d}\right\} \text { PureFSO }}
\end{gathered}
$$

where $E_{\text {saving }}($ Week) is the energy saved in a particular week by the proposed dual-rate, dual-link hybrid architecture. $E_{10 G}(F S O)$ and $E_{10 G}(S M F)$ are the energy consumed by the FSO link and SMF link, respectively, for transmission of broadband signal at $10 \mathrm{Gbps}$. Similarly, $E_{l G}(F S O)$ and $E_{l G}(S M F)$ are the energy consumed by the FSO link and SMF link respectively for transmission of narrowband signal at $1 \mathrm{Gbps}$. $d$ denotes the days ranging from 1 (Monday) to 7 (Sunday). $P_{10 G}$ and $P_{1 G}$ denote the power consumption at the OLT during transmission of broad band and narrow band signals, respectively. This OLT power consumption $\left(P_{10 G}\right.$ and $\left.P_{1 G}\right)$ occurs due to two factors. The first one is fixed and is required to drive the line card holding the TRx $(3.5 \mathrm{~W}$ for $10 \mathrm{G}$ line card and $0.5 \mathrm{~W}$ for 1 G line card) (Tadokoro et al. 2012) and the second one is required to drive the TRx itself for transmitting the data to the link. This TRx power is used to compensate for the link loss and to provide sufficient power at the receiver to meet the receiver sensitivities. Therefore, the TRx power is dependent on the weather conditions and link whether it is SMF or FSO.

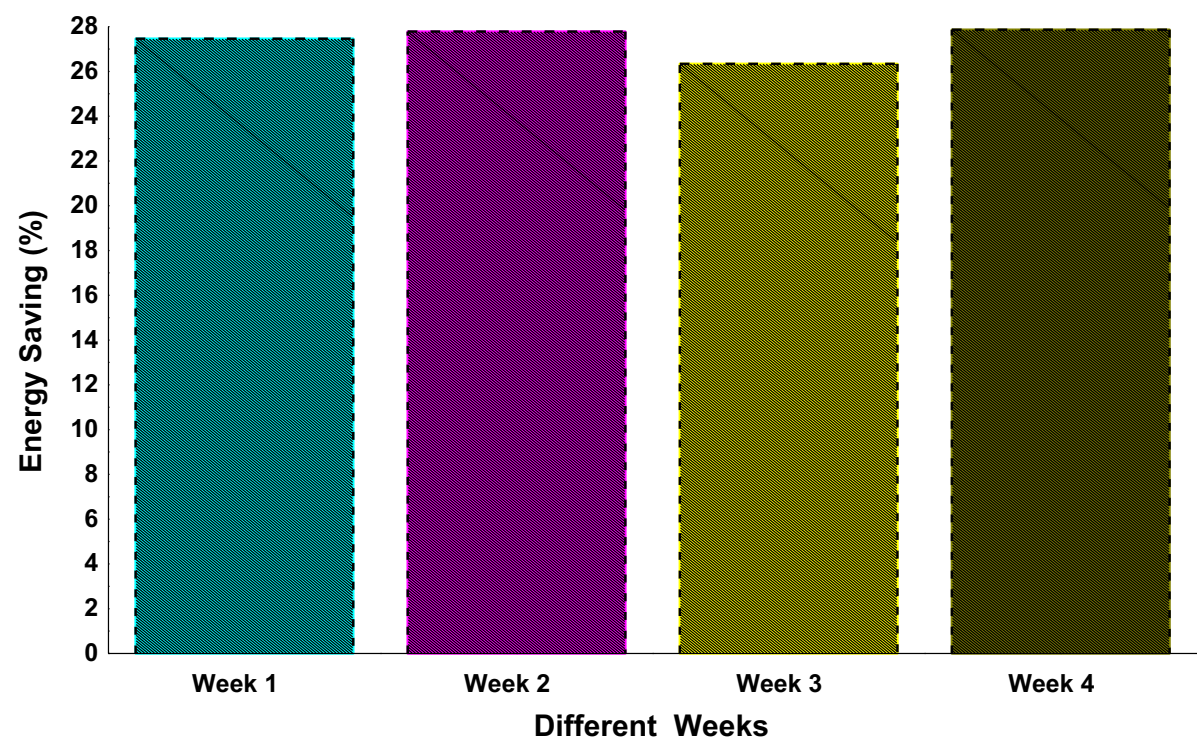

Fig. 11 Energy saving (\%) of proposed architecture w.r.t. pure FSO link for different weeks of a month 
The 10G TRx requires more power as compared to $1 \mathrm{G}$ TRx. $h_{10 G}$ and $h_{1 G}$ are the time duration for which $10 \mathrm{G}$ TRx and $1 \mathrm{G}$ TRx that are used each day.

Based on Eqs. (9)-(12) for considered arbitrary environmental conditions (as given in Table 3), the energy-saving for each week of a month by using the proposed dual-rate hybrid FSO/SMF architecture as compared to the pure FSO link, is approximately $27 \%$ and is given in Fig. 11. The average absolute energy saving per week is almost $171 \mathrm{~W}$. This small amount of energy-saving per week allows us to reduce the energy consumption of approximately $8.2 \mathrm{~kW}$ in the entire year for a single network and $8.2 \times \mathrm{N} \mathrm{kW}$ for $\mathrm{N}$ networks. The energy-saving may vary for different environmental circumstances. However, the developed dual-rate/dual-link based hybrid architecture provides an excellent way to reduce energy consumption significantly.

Energy-saving of the proposed architecture w.r.t the pure SMF link for different weeks are also calculated and given below by the following equations:

$$
E_{\text {Saving }}(\text { Week })=\sum_{d=1}^{7}\left[\begin{array}{l}
\left\{E_{10 G}(S M F)_{d}+E_{1 G}(S M F)_{d}\right\} \text { Pure SMF } \\
\left.-\left\{E_{10 G}(F S O)_{d}+E_{1 G}(F S O)_{d}+E_{10 G}(S M F)_{d}+E_{1 G}(S M F)_{d}\right\} \text { Hybrid }\right]
\end{array}\right]
$$

$$
\begin{aligned}
& E_{\text {Saving }}(\text { Week }) \\
& =\sum_{d=1}^{7}\left\{P_{10 G}(S M F)_{d} \cdot h_{10 G}(S M F)_{d}+P_{1 G}(S M F)_{d} \cdot h_{1 G}(S M F)_{d}\right\} \\
& \text { PureSMF }-\left\{P_{10 G}(F S O)_{d} \cdot h_{10 G}(F S O)_{d}+P_{1 G}(F S O)_{d} \cdot h_{1 G}(F S O)_{d}+P_{10 G}(S M F)_{d} \cdot h_{10 G}(S M F)_{d}+P_{10 G}(S M F)_{d} \cdot h_{10 G}(S M F)_{d}\right\} \text { Hybrid }
\end{aligned}
$$

$$
E_{\text {Saving }}(\%)=1-\frac{\sum_{d=1}^{7}\left\{E_{10 G}(F S O)_{d}+E_{1 G}(F S O)_{d}+E_{10 G}(S M F)_{d}+E_{1 G}(S M F)_{d}\right\} \text { Hybrid }}{\sum_{d=1}^{7}\left\{E_{10 G}(S M F)_{d}+E_{1 G}(S M F)_{d}\right\} \text { PureSMF }}
$$

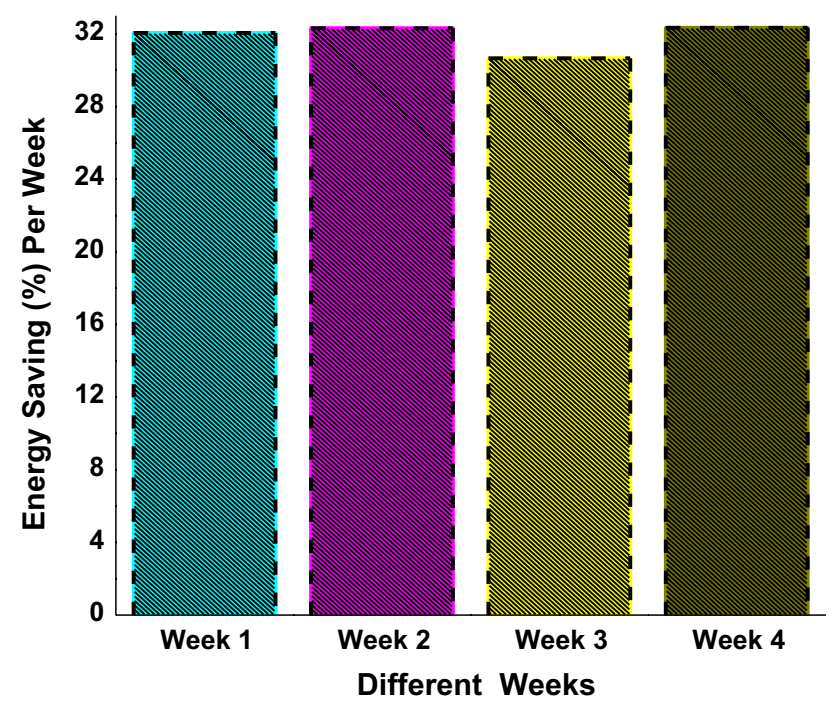

Fig. 12 Energy saving (\%) of proposed architecture w.r.t. pure SMF link for different weeks of a month 


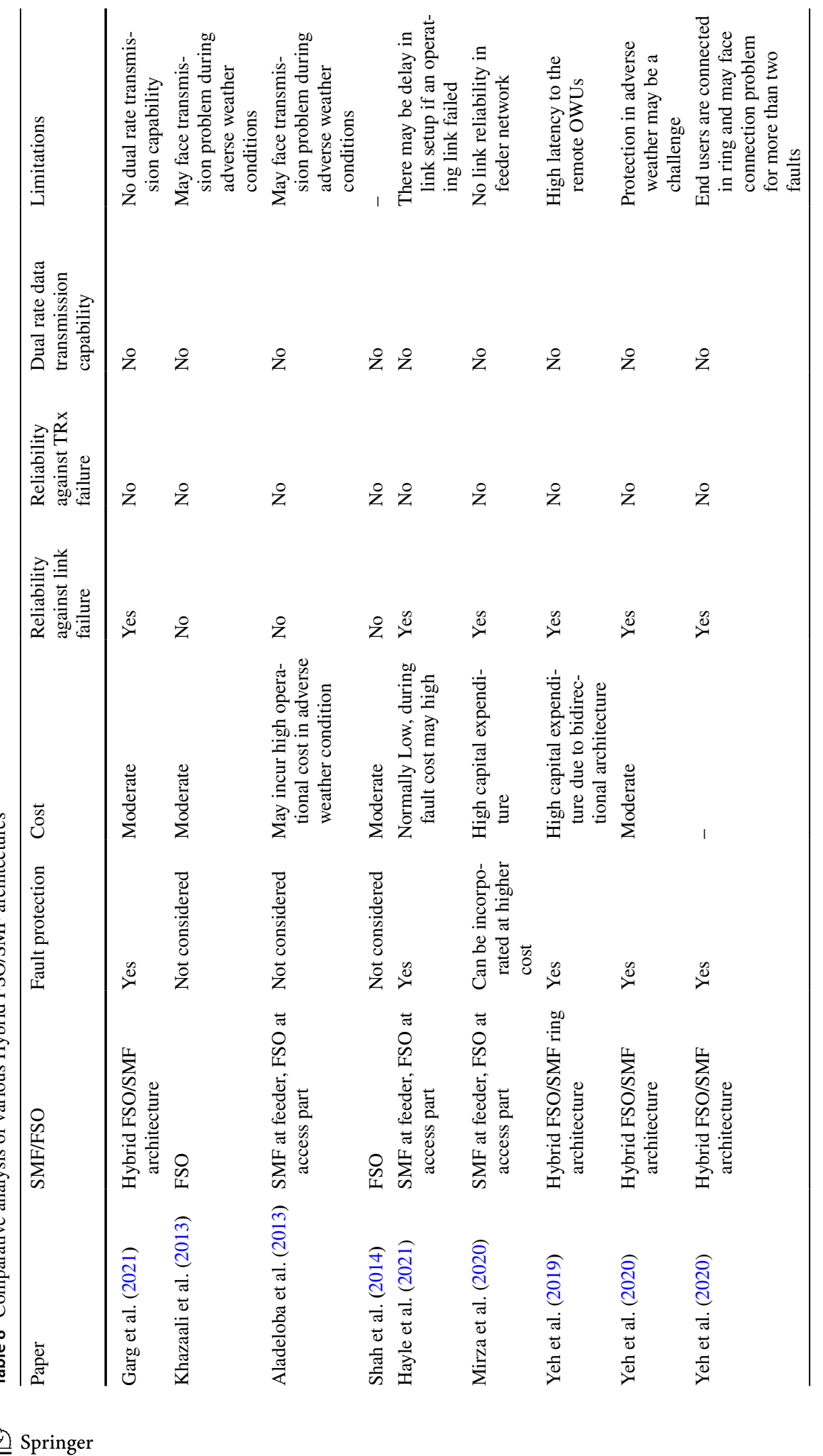




$$
E_{\text {Saving }}(\%)=1-\frac{\sum_{d=1}^{7}\left\{P_{10 G}(S M F)_{d} \cdot h_{10 G}(S M F)_{d}+P_{1 G}(S M F)_{d} \cdot h_{1 G}(S M F)_{d}\right\} \text { Hybrid }}{\sum_{d=1}^{7}\left\{P_{10 G}(S M F)_{d} \cdot h_{10 G}(S M F)_{d}+P_{1 G}(S M F)_{d} \cdot h_{1 G}(S M F)_{d}\right\} \text { PureSMF }}
$$

where each term has similar meaning as mentioned for Eqs. (9)-(12). Based on Eqs. (13) - (16) for given network conditions, the proposed dual-rate hybrid FSO/SMF architecture as compared to the pure SMF link, is approximately $31.84 \%$ and is given in Fig. 12. The average absolute energy saving per week is almost $212.25 \mathrm{~W}$, which is significant for a network.

The various advantages of the proposed dual-rate hybrid FSO/SMF architecture as compared to the pure FSO link with its limitations are illustrated in Table 8.

\section{Conclusion}

A dual-rate/dual-link hybrid SMF/FSO integrated optical access network is proposed which is capable of transmitting the data at a very high speed ranging from 1 to $20 \mathrm{Gbps}$ depending upon the traffic load. The proposed architecture is well suited for clear as well as adverse weather conditions. For clear weather conditions, the FSO link is preferred in order to take the advantages of transmission without spectrum licensing, low nonlinear effects, and no link faults during maintenance of the roads etc. The FSO links also have very low installation and maintenance time and legacy networks can be extended hasslefree in the hilly, mountain and river areas etc., where there are lots of challenges to extend the fiber optic links.

In case of adverse weather conditions or link failure and on the fiber optic link availability, services can be continued on the SMF simply by tuning the transmitted wavelength by $50 \mathrm{GHz}$. The proposed architecture is reliable against the link failure and has $100 \%$ higher reliability as compared to the pure FSO network. This architecture is also reliable against TRx failure, because in case of failure of 1 Gbps TRx the services remain continued on 10 Gbps TRx and vice versa. The BER performance of both the links SMF/FSO for narrowband and broadband transmission has been verified and are quite acceptable for both the links. However there is approximately $0.6 \mathrm{~dB}$ FSO link power penalty at the receiver as compared to the pure SMF link. For hybrid SMF/FSO integrated architecture, the FSO link power penalty reduces to $0.3 \mathrm{~dB}$. For integrated link, approximately $27 \%$ of the energy 


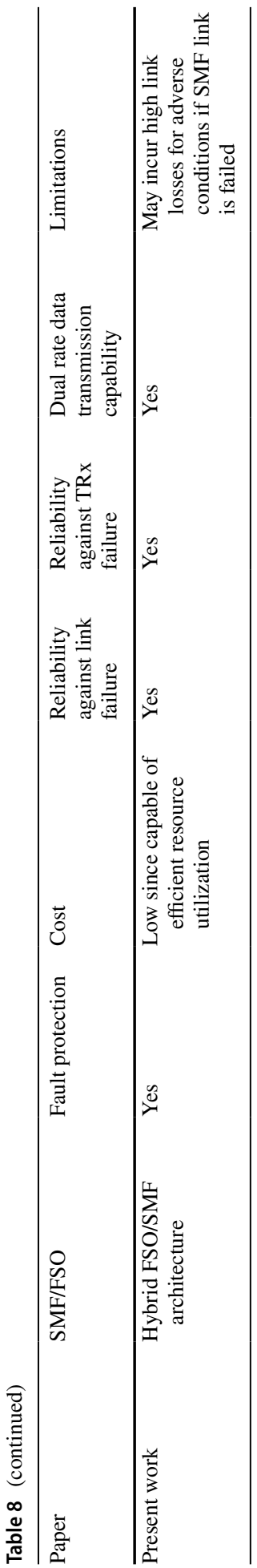

글 Springer 
consumption is reduced as compared to the pure FSO link. The average absolute energy saving per year for a network is approximately $8.2 \mathrm{~kW}$. The proposed link also reduces the energy consumption of approximately $\sim 31.84 \%$ as compared to pure SMF link. The proposed architecture strengthens the existing ICT infrastructure and is responsible for supporting the development of smart cities that handle high-speed internet services.

For higher phase noise, the performance of both FSO and SMF transmission will degrade and the quality factor of the received optical signal will be deteriorated. Alternatively, the bit error rate performance of the received signal will be degraded. With the higher phase noise, the spectral efficiency of the channel will be very poor. This needs more investigation, which we intend to do in a future work.

Acknowledgements The corresponding author would like to acknowledge the THz Communication and Photonics Lab, Department of ECE, Malaviya National Institute of Technology, Jaipur for providing the simulation facilities.

\section{References}

Agrawal, G.P.: Nonlinear Fiber Optics, 5th edn. Academic Press, NY, USA (2013)

Aladeloba, A.O., Woolfson, M.S., Phillips, A.J.: WDM FSO network with turbulence-accentuated interchannel crosstalk. IEEE/OSA J. Opt. Commun. Netw. 5(6), 641-651 (2013). https://doi.org/10.1364/JOCN.5.000641

Alkholidi, A.G., Altowij, K.S.: Free space optical communications - theory and practices. Contemp. Issues Wirel Commun. (2014). https://doi.org/10.5772/58884

Badar, N., Jha, R.K.: Performance comparison of various modulation schemes over free space optical (FSO) link employing Gamma-Gamma fading model. Opt. Quant. Electron. 49, 192 (2017). https://doi.org/ 10.1007/s11082-017-1025-4

Badar, N., Jha, R.K., Towfeeq, I.: Performance analysis of an $80(8 \times 10)$ Gbps RZ-DPSK based WDMFSO system under combined effects of various weather conditions and atmospheric turbulence induced fading employing Gamma-Gamma fading model. Opt. Quant. Electron. 50, 44 (2018). https://doi.org/10.1007/s11082-017-1306-y

Chae, C.J., Tucker, R.S.: Passive virtual optical networking technique using a cyclic N $\times \mathrm{N}$ arrayed waveguide grating for multiple multihop ring networks. IEEE Photonics Technol. Lett. 16(3), 948-950 (2004). https://doi.org/10.1109/LPT.2004.823742

Chan, V.W.S.: Free-space optical communications. J. Lightwave Technol. 24(12), 4750-4762 (2006). https://doi.org/10.1109/JLT.2006.885252

Ciaramella, E., Arimoto, Y., Contestabile, G., Presi, M., D’Errico, A., Guarino, V., Matsumoto, M.: 128 terabit/s $(32 \times 40 \mathrm{Gbit} / \mathrm{s}) \mathrm{wdm}$ transmission system for free space optical communications. IEEE J. Sel. Areas Commun. 27(9), 1639-1645 (2009). https://doi.org/10.1109/JSAC.2009.091213

Elsayed, E.E., Yousif, B.B.: Performance enhancement of M-ary pulse-position modulation for a wavelength division multiplexing free-space optical systems impaired by interchannel crosstalk, pointing error, and ASE noise. Opt. Commun. 475, 1-9 (2020). https://doi.org/10.1016/j.optcom.2020.126219

Garg, A.K., Janyani, V., Batagelj, B.: Ring based latency-aware and energy-efficient Hybrid WDM TDM-PON with ODN interconnection capability for smart cities. Opt. Fiber Technol. (2020). https://doi.org/10.1016/j.yofte.2020.102242

Garg, A.K., Janyani, V., Batagelj, B., Abidin, N.H.Z., Bakar, M.A.: Hybrid FSO/fiber optic link based reliable \& energy efficient WDM optical network architecture. Opt. Fiber Technol. 61, 102422 (2021). https://doi.org/10.1016/j.yofte.2020.102422

Hayle, S.T., Manie, Y.C., Shao, G.M., Chiu, P.H., Yeh, T.Y., You, S.L., Peng, P.C.: Integration of fiber and FSO network with fault-protection for optical access network. Opt. Commun. 484, 1-5 (2021). https://doi.org/10.1016/j.optcom.2020.126676

Horn, W., Kroesen, S., Herrmann, J., Imbrock, J., Denz, C.: Electro-optical tunable waveguide Bragg gratings in lithium niobate induced by femtosecond laser writing. Opt. Express 20, 26922-26928 (2012)

https://www.corning.com/opcomm/oem-solutions/worldwide/en/products/micro-optics-products/wavel ength-management.html. Accessed from 20 Feb 2021

https://www.fs.com/c/wdm-optical-access-1. Accessed from 20 Feb 2021

https://www.optcore.net/product/. Accessed from 25 Mar 2021 
Khalighi, M.A., Uysal, M.: Survey on free space optical communication: a communication theory perspective. IEEE Commun. Surv. Tutor. 16(4), 2231-2258 (2014). https://doi.org/10.1109/COMST. 2014.2329501

Khazaali, M.: Performance of a 162.5 Gbps WDM system transmitting over FSO medium. In: Proceeding of the 2013 IEEE 4th International Conference on Photonics (ICP), 84-86 (2013). https://doi. org/10.1109/ICP.2013.6687075

Kramer, A., Kramer, K.Z.: The potential impact of the Covid-19 pandemic on occupational status, work from home, and occupational mobility. J. Vocat. Behav. 119, 103442 (2020). https://doi.org/10. 1016/j.jvb.2020.103442

Leijtens, X.J.M., Kuhlow, B., Smit, M.K.: Arrayed waveguide gratings. In: Venghaus, H. (ed.) Wavelength Filters in Fiber Optics, pp. 125-187. Springer, New York (2006)

Liaw, S.K., Hsu, K.Y., Yeh, J.G., Lin, Y.M., Yu, Y.L.: Impacts of environmental factors to bi-directional $2 \times 40 \mathrm{~Gb} / \mathrm{s}$ WDM free-space optical communication. Opt. Commun. 396, 127-133 (2017). https:// doi.org/10.1016/j.optcom.2017.03.042

Malik, A., Singh, P.: Free space optics: current applications and future challenge. Int. J. Opt. (2015). https://doi.org/10.1155/2015/945483

Mirza, J., Imtiaz, W.A., Aljohani, A.J., Atieh, A., Ghafoor, S.: Design and analysis of a $32 \times 5$ Gbps passive optical network employing FSO based protection at the distribution level. Alex. Eng. J. 59(6), 4621-4631 (2020). https://doi.org/10.1016/j.aej.2020.08.020

Mohanty, S.P., Choppali, U., Kougianos, E.: Everything you wanted to know about smart cities: the Internet of things is the backbone. IEEE Consum. Electron. Mag. 5(3), 60-70 (2016). https://doi. org/10.1109/MCE.2016.2556879

Shah, D., Kothari, D.: Optimization of 2.5 Gbps WDM-FSO link range under different rain conditions in Ahmedabad. In: 2014 Annual IEEE India Conference (INDICON), 1-4, (2014). https://doi.org/10. 1109/INDICON.2014.7030643

Tadokoro, M., Kubo, R., Nishihara, S., Yamada, T., Nakamura, H.: Adaptive bandwidth aggregation mechanisms using a shared wavelength for energy-efficient WDM/TDM-PON. In: in Proceedings of the 10th International Conference on Optical Internet, Yokohama, Kanagawa, Japan, 87-88, (2012)

Toyoda, S., Ooba, N., Kitoh, T., Kurihara, T., Maruno, T.: Wide tuning range and low operating power AWG-based thermo-optic wavelength tunable filter using polymer waveguides. Electron. Lett. 37(18), 1130-1132 (2001)

Vu, B.T., Dang, N.T., Thang, T.C., Pham, A.T.: Bit error rate analysis of rectangular QAM/FSO systems using an APD receiver over atmospheric turbulence channels. J. Opt. Commun. Netw. 5(5), 437-446 (2013)

Yamamoto, S., Mochizuki, K., Wakabayashi, H., Iwamoto, Y.: Long-haul high-speed optical communication systems using a semiconductor laser amplifier. J. Lightwave Technol. 6(10), 1554-1558 (1988). https://doi.org/10.1109/50.7915

Yeh, C., Guo, B., Gu, C., Chow, C., Lin, W.: Use of same WDM channels in fiber network for bidirectional free space optical communication with Rayleigh backscattering interference alleviation. IEEE Access 7, 169571-169576 (2019). https://doi.org/10.1109/ACCESS.2019.2954951

Yeh, C.H., Hsu, W.H., Wang, B.Y., You, W.Y., Chen, J.R., Chow, C.W., Kuei, S.: Fiber- and fso-protected connections for long-reach TWDM access architecture with fault protection. IEEE Access 8, 189982189988 (2020). https://doi.org/10.1109/ACCESS.2020.3031609

Yeh, C., Xie, Y., Luo, C., Chow, C.: Integration of FSO traffic in ring-topology bidirectional fiber access network with fault protection. IEEE Commun. Lett. 24(3), 589-592 (2020). https://doi.org/10.1109/ LCOMM.2019.2960221

Yu, Y., Liaw, S., Chou, H., Le-Minh, H., Ghassemlooy, Z.: A hybrid optical fiber and fso system for bidirectional communications used in bridges. IEEE Photonics J 7(6), 1-9 (2015). https://doi.org/10.1109/ JPHOT.2015.2488286

Publisher's Note Springer Nature remains neutral with regard to jurisdictional claims in published maps and institutional affiliations. 


\section{Authors and Affiliations}

\section{Amit Kumar Garg ${ }^{1}$. Sanjeev Kumar Metya ${ }^{2} \cdot$ Ghanshyam Singh $^{3} \cdot$ Vijay Janyani $^{3}$. Moustafa H. Aly ${ }^{4}$ N. H. Zainol Abidin ${ }^{5}$}

1 Department of Electronics and Communication Engineering, Indian Institute of Information Technology, Kota, (MNIT Campus), Jaipur, India

2 Department of ECE, National Institute of Technology Arunachal Pradesh, Jote, India

3 Department of ECE, Malaviya National Institute of Technology, Jaipur, India

4 Department of ECE, College of Engineering and Technology, Arab Academy for Science, Technology and Maritime Transport, Alexandria, Egypt

5 Faculty of Engineering, Department of Computer and Communication Systems Engineering, Universiti Putra Malaysia, UPM, 43400 Serdang, Selangor, Malaysia 